\title{
MODELO DE EVALUACIÓN EN LA INTERVENCIÓN \\ DE ESPACIOS COMERGIALES, \\ Bajo criterios de construcción sustentable
}

\author{
EVALUATION MODEL IN COMMERGIAL \\ SPACES INTERVENTIONS, \\ Under sustainable construction criteria
}

\author{
Verónica Lorena Vélez Jaramillo \\ Investigador Independiente \\ Ecuador
}

Magíster en Diseño de Espacios Interiores en la Universidad del Azuay; Magíster en Retail Design, Diseño de Espacios Comerciales, Escuela de Diseño Elisava de la Universidad Pompeu Fabra, Barcelona, España (2012); Posgrado en Iluminación Arquitectónica en la Pontificia Universidad Católica de Chile (2014); Diseñadora de Espacios Interiores (2010) en la Universidad del Azuay.

verovelezj@gmail.com

orcid.org/0000-0001-5092-4883 


\section{Carlos Esteban Contreras Lojano}

Universidad del Azuay

Ecuador

Arquitecto, Universidad de Cuenca (2005); Magíster en Proyectos Arquitectónicos, Universidad de Cuenca (2009); Magíster en Construcciones, Universidad de Cuenca (2013); Profesor Titular de la Facultad de Artes (Escuela de Diseño) de la Universidad de Cuenca, 2007 -2015; Profesor Titular de Facultad de Diseño (Escuela de Diseño de Interiores) en la Universidad del Azuay, desde 2013 hasta la actualidad; Primer puesto en el Concurso Nacional de Anteproyectos para el Barranco de la ciudad de Cuenca; Primer puesto nacional en Proyectos de Unidades Educativas del Milenio, "Escuela Marieta de Veintimilla", Motupe - Loja; Socio fundador de la Constructora Contreras Construhermanos Cltda, abril 2015 - actualidad.

arqcarloscontreras@gmail.com

orcid.org/0000-0001-6029-2533

Fecha de recepción: 17 de septiembre, 2020. Aceptación: 16 de abril, 2021. 


\section{Resumen}

Los problemas ambientales, en nuestro tiempo, son el efecto de un proceso lineal insustentable de producción, explotación de recursos y generación de residuos que están agotando la naturaleza. Por ello, tomar medidas urgentes es de suma importancia.

La presente investigación pretende desarrollar un modelo de evaluación que nos lleve a identificar y cuantificar el consumo energético y emisión de $\mathrm{CO}_{2}$ de los materiales utilizados en propuestas de diseño interior en espacios comerciales, principalmente restaurantes y cafeterías de la ciudad de Cuenca, Ecuador. Esta investigación se aplicará en tres intervenciones de remodelación comercial para: determinar el grado de impacto ambiental y comparar con otras soluciones y, así, tomar decisiones acertadas al momento de desarrollar los proyectos.

El modelo de evaluación que se propone, para cuantificar el consumo energético y emisión de $\mathrm{CO}_{2}$ de los materiales derivados de su fabricación, no incluye transporte ni puesta en obra. Este será una herramienta que permita desarrollar propuestas sustentables, con disminución de energía consumida y emisiones de $\mathrm{CO}_{2^{\prime}}$ al sustituir los materiales utilizados por otros con menor impacto.

\section{Palabras clave}

Economía circular, diseño, huella de carbono, indicadores ambientales, metodología.

\section{Abstract}

Environmental problems of our time are the result of a linear, unsustainable process of production, exploitation of resources, and generation of waste, which is exhausting nature. Therefore, taking urgent action is of the utmost importance. This research aims to develop an evaluation model to identify and quantify the materials used in interior design projects for commercial spaces, mainly restaurants and cafeterias, in the city of Cuenca, Ecuador. This research will be applied in three commercial remodeling interventions, to determine the degree of environmental impact and compare it with other solutions and thus, make the right decisions when using them. The proposed evaluation model to quantify the energetic consumption and $\mathrm{CO}_{2}$ emissions of the materials derived from its manufacture does not include transport or commissioning. This tool will allow the development of sustainable proposals, managing to reduce the amount of energy consumed and $\mathrm{CO}_{2}$ emissions from the manufacturing process, by replacing the materials used with others with less impact.

\section{Keywords}

Circular economy, design, carbon footprint, environmental indicators, methodology. 


\section{Introducción}

Esta investigación surge de la toma de conciencia, desde nuestro actuar como diseñadores de espacios interiores, de la necesidad de disminuir el impacto ambiental causado por el sector de la construcción, del cual somos parte. "El modelo productivo dominante, que caracteriza a la producción de la arquitectura, puede sintetizarse en la secuencia lineal extracción, fabricación, uso y residuo" (Wadel, Avellaneda y Cuchí, 2010, p. 38).

Estamos agotando la naturaleza a un ritmo mayor que el de su regeneración, por la acelerada explotación de recursos y generación de residuos. Otros materiales utilizados en proyectos de arquitectura y diseño, procedentes de procesos industriales, tienen gran cantidad de energía incorporada que, también, son extraídos de la naturaleza de manera destructiva y generan residuos tóxicos que contaminan, de forma grave, el medio ambiente.

Aunque los arquitectos y diseñadores no tengamos control sobre los procesos de fabricación de materiales, podemos desempeñar un papel importante en la reducción de su impacto al momento de seleccionarlos. La materialización de proyectos es la acción fundamental que causa este impacto y puede incrementar sus efectos por falta de optimización de los mismos (Serrano, Quesada, López, Guillen, y Orellana, 2015).

De esta manera, el modelo de evaluación propuesto en esta investigación para intervenciones de espacios comerciales, bajo criterios de construcción sustentable, permite analizar y seleccionar elementos de construcción que utilicen menos recursos materiales, y buscar alternativas menos agresivas al medio ambiente, ya que los residuos son generados en todas las etapas del ciclo de vida de un determinado material.

El objeto de esta investigación, que se desarrollará en tres intervenciones de diseño interior comercial, es: establecer un modelo de evaluación que cuantifique las emisiones de $\mathrm{CO}_{2}$ y la energía consumida en el proceso de fabricación de materiales que serán empleados en las intervenciones de remodelación, con la finalidad de medir el grado de impacto ambiental de los proyectos, en pro de buscar alternativas que permitan desarrollar propuestas igual de sustentables pero que favorezcan al medioambiente y bienestar de los usuarios.

En países de Latinoamérica, como Ecuador, apenas se ha incursionado en prácticas sustentables en la construcción, debido a la falta de desarrollo industrial, personal técnico, apoyo del sector público y privado, y otras barreras comunes en el país, por las cuales se sigue construyendo edificios en forma convencional y generando impactos ambientales (...) En Ecuador, cerca del $9 \%$ del producto interno bruto (PIB), un valor significativo, corresponde a la construcción (Daza, 2010, pp. 2,6).

\section{Proceso investigativo}

\section{Complejidad ambiental}

La Complejidad Ambiental es entendida como la crisis ambiental de nuestro tiempo que, ante todo, es el resultado de las formas de conocimiento, a través de las cuales la humanidad ha construido el mundo y lo ha destruido. Esta racionalidad dominante encubre la complejidad ambiental, negándola en un mundo economizado, sujeto por un proceso incontrolable e insustentable de producción (Leff , 2007, sn).
"Es por esto que nos vemos llevados a replantear el problema del desarrollo, rechazando la noción tan grosera y tan bárbara que ha reinado largo tiempo" (Morin, 1996, s.n).

El sistema económico es lineal y finito, pues utiliza los recursos de la Naturaleza y le devuelve desechos, procedentes de su actividad y, como su nombre lo indica, no son de utilidad a la naturaleza y la daña de forma irreparable. Cuando en la naturaleza el proceso es cíclico e infinito, pues los recursos que se utilizan se convierten en nuevos re- 
cursos para alimentar otros organismos, no existe el concepto de 'residuo', ni el concepto de 'daño medioambiental (Garrido, s.f, p. 8).

Sin duda, pasar de un proceso lineal y finito a un proceso cíclico e infinito, que integra la naturaleza, no va a ser fácil, pero es de suma urgencia empezar a hacerlo. Y ese es el objetivo del denominado "desarrollo sustentable".

Para Wackernagel y Rees (1996) no se puede pensar que los emprendimientos humanos estén separados del mundo natural, porque tal separación no existe. Es importante entender que no sólo estamos conectados con la naturaleza sino que somos la naturaleza. Es así que, si los humanos somos parte de la naturaleza el medio ambiente ya no es más el telón de fondo sino que es la obra misma. La humanidad depende de la naturaleza y no a la inversa.

La actividad humana siempre ha ocasionado algún tipo de daño al medio ambiente, y más aún en los últimos años, con la desmesurada actividad industrial que, poco a poco, ha ido deteriorando el medioambiente. Sin duda alguna es necesario tomar acciones para frenar el daño causado (Garrido, s.f). Los principales problemas actuales son planetarios y representan una amenaza para la humanidad; así que, de todos, en el que hay que enfocarse es en el ecológico, que es de carácter holístico, lo que sucede en una parte del mundo tiene un alcance planetario (Morin, 1996).

\section{Desarrollo sustentable}

La introducción de los problemas ambientales en el ámbito político tuvo lugar a finales de los sesenta e inicio de los setenta y se convirtió en alarma que despertó al mundo, en cuanto al notable daño que causamos a diario a la naturaleza, sugiriendo un cambio en el modo de vida para evitar el sufrimiento humano y el daño al medio ambiente. Esto se conoció gracias al documento desarrollado por un grupo de trabajo, conocido como Informe Brundtland, en abril de 1987 (Boada y Toledo 2003, en López, López-Hernández y Ancona, 2005, s.n).

Fue en 1987 que se empezó a hablar del concepto de "desarrollo sustentable", entendido como aquel desarrollo que, sin agotar los recursos ni dañar el medioambiente, permite satisfacer las necesidades presentes sin comprometer las futuras. No se pretende la no utilización de recursos sino un uso coherente de los mismos. (López, et al., 2005, s.n).

El desarrollo sustentable no deja de ser desarrollo, la diferencia es que tiene un calificativo con particularidades y características sustentables, es decir que puede desenvolverse y permanecer para futuras generaciones sin generar impactos negativos al medio ambiente.

\section{Economía circular}

El sistema lineal de producción y consumo sigue vigente desde la revolución industrial y se basa en la extracción de materia prima, producción de bienes, consumo y generación de residuos (Morató, Jiménez y Tollin, 2017). Pero no es un sistema sostenible ni compatible con un mundo de recursos limitados por su limitada capacidad de carga.

La economía circular redirecciona los sistemas de producción y consumo actuales hacia un cambio radical de sistemas, que sean regenerativos y cíclicos a partir de su diseño, para, de esta manera, optimizar los recursos y la energía; disminuyendo la creación de residuos e impactos negativos para el medioambiente, clima y salud humana. "En la actualidad la mayor parte de las empresas ecuatorianas y extranjeras, que operan en el país, siguen el modelo económico lineal, que no ha cambiado desde la primera revolución industrial" (Arroyo, Bravo y Rivera, 2018, p. 140). 
Figura 1. Economía circular

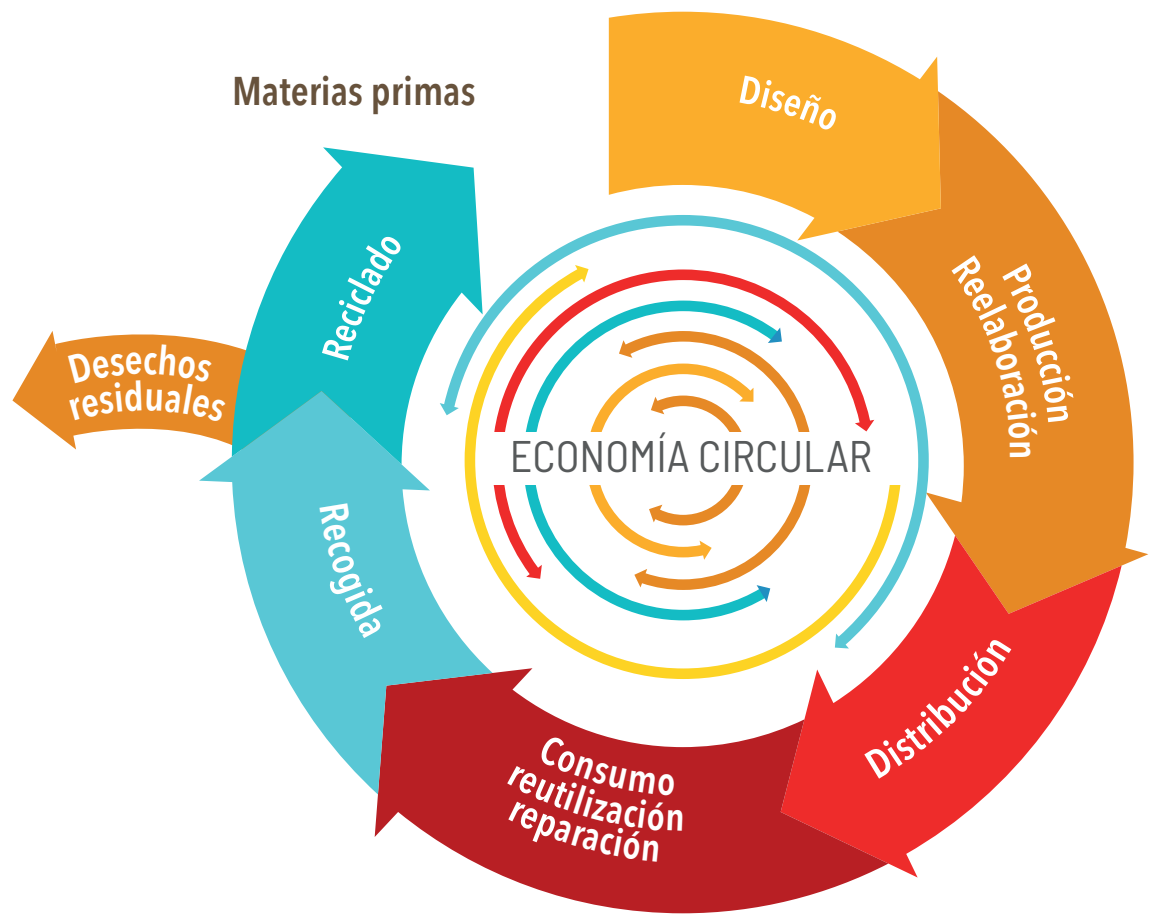

De Asociación Sostenibilidad y Arquitectura, ASA, (2017).

Datos revelados por el Instituto Nacional de Estadísticas y Censos (INEC) indican que, en el Ecuador, $80 \%$ de empresas no invierten en protección ni reducción del impacto ambiental y solo $2 \%$ han incluido sistemas de gestión ambiental.

\section{Capacidad de carga de la tierra}

El desarrollo económico y tecnológico de las sociedades ha dependido, por mucho tiempo, de la energía. "Es un recurso demandado para satisfacer las necesidades de producción y proporcionar bienestar. En la antigüedad se dependía de la fuerza muscular de hombres y animales para satisfacer estas necesidades, hasta que aparecieron los combustibles fósiles, carbón, petróleo y gas" (Morillón y Hernández, 2011, p. 1).
La combustión de estos recursos produce emisión de gases a la atmósfera, como el dióxido de carbono $\left(\mathrm{CO}_{2}\right)$, principal gas de efecto invernadero (GEI) y su exceso está contribuyendo al calentamiento de la Tierra, cuya capacidad para eliminar y regenerar los subproductos derivados de las actividades humanas del planeta es limitada, como reconoció la Comisión Brundtland en 1987.

La demanda de energía que requieren los materiales de construcción en todas las etapas: extracción, transporte y fabricación, proviene de combustibles de origen fósil, que son responsables de producir y arrojar a la atmósfera emisiones tóxicas como $\mathrm{CO}_{2}$. Estas emisiones superan la capacidad de absorción de los sumideros de carbono de la Tierra (bosques y océanos) y contribuyen, de manera importante, al efecto invernadero y al deterioro de nuestro planeta (Morillón y Hernández, 2011). 
Un informe presentado por la ONU muestra que el sector constructivo consume $40 \%$ de toda la energía; 39\% del dióxido de carbono $\left(\mathrm{CO}_{2}\right)$ es emitido a la atmósfera; la extracción de hasta $30 \%$ de las materias primas en el entorno; $25 \%$ de los residuos sólidos generados provienen de actividades ligadas a la construcción y, por último, consume hasta $25 \%$ de agua y ocupa, aproximadamente, $12 \%$ de la tierra. "Estos datos son un signo de alarma, no para evitar las actividades constructivas sino para buscar la manera de minimizar los impactos sobre el medio ambiente" (García, Quito y Perdomo, 2019, s.n).

\section{Impacto ambiental de los materiales de construcción}

Dentro de la arquitectura y el diseño, el concepto sustentable, generalmente, basa su atención en proyectar espacios operacionalmente eficientes, enfocados en reducir el uso de energía 0 emisiones operacionales de los edificios y dejan de lado otros factores, como la selección de materiales que son, muchas veces, extraídos destructivamente de la naturaleza y son responsables de grandes emisiones tóxicas, derivados de su proceso de fabricación o de su comportamiento como residuo. La construcción sustentable no debe cambiar el concepto de construcción tradicional, simplemente, en todas las etapas del proyecto, se debe actuar conscientemente y con responsabilidad con el medio ambiente.

La aparición de medios de extracción y fabricación más eficientes, así como un transporte mucho más globalizado por la abundante y barata disponibilidad de energía, hace que la producción de materiales pierda la inmediatez de lo cercano y se convierta en una actividad altamente impactante (Corral, 2017, s.n).

En respuesta a esta problemática actual surgen los conocidos materiales sustentables, como aquellos elaborados con energía renovable, sea solar, eólica o hidráulica; materiales de origen local, que provienen de empresas consientes con el medio am- biente, que desarrollan prácticas respetuosas o que, para obtener nuevos materiales, utilizan materia prima reciclada. De igual manera, contribuye a reducir daños ambientales el análisis de energía en la elaboración de los materiales (establecida como energía incorporada $\mathrm{MJ} / \mathrm{Kg}$, Mega Joules de energía que se utiliza para un kilogramo de material) y las emisiones de $\mathrm{CO}_{2}$ procedentes del proceso de fabricación.

No existen materiales cien por ciento sustentables, pero sí aquellos que procuran reducir los impactos al medio ambiente y que los podemos identificar a través de las Declaraciones Ambientales de Producto (DAP) y el eco-etiquetado, que proporcionan información objetiva referente a su fabricación y que permiten poder evaluarlos. Las DAP, 0 por sus siglas en inglés EPD, son una herramienta muy importante que permite identificar el comportamiento ambiental de un material y sirve como una herramienta fundamental para conseguir propuestas más sustentables.

\section{Indicadores ambientales}

Un indicador ambiental es una variable 0 estimación ambiental (emisiones de $\mathrm{CO}_{2}$ ), interpretada como una propiedad física, que debe ser medida y que proporciona información agregada y sintética sobre un fenómeno (cambio climático), es decir es una herramienta para medir esa propiedad (Macías y Navarro, 2010, p. 90).

Para Bermejo (2001), en la gestión ambiental los indicadores son un medio para simplificar una realidad compleja y se utilizan para poder evaluar los problemas, establecer objetivos y controlar el cumplimiento de dichos objetivos (citado en Paternoster, 2011).

\section{Certificaciones ambientales}

Para Lajara (2012) es importante tomar conciencia sobre los beneficios que tienen los proyectos sustentables, no solo para el planeta sino 
también para los usuarios. Por ello, existen una serie de certificaciones que se conceden cuando los proyectos reúnen algunos requisitos fundamentales de construcción sustentable y que puntúan al edificio en función de las especificaciones definidas.

A pesar de que la mayoría de construcciones se siguen realizando sin criterios de sostenibilidad, cada vez más se está tomando conciencia de la importancia del cuidado del medioambiente.

Y como respuesta, desde el año 2000, los métodos de evaluación medioambiental para edificios han aumentado de manera significativa.

BREEAM (Building Research Establishment Environmental Assessment Methodology) fue el primer sistema (1990) y el de más trayectoria, que ofreció un método de etiquetado de edificios, aunque LEED (Leadership in Energy and Environmental Design) es el de mayor implantación en el mercado de grandes edificios y el más difundido a nivel internacional (Macías y García, 2010, s.n).

VERDE (acrónimo de Valoración de Eficiencia de Referencia De Edificios), por otro lado, es la certificación española que ayuda a profesionales de la construcción a conseguir edificios con menor impacto medioambiental. En lo que se refiere al impacto de los materiales de la construcción en edificios, LEED propone el aumento de uso de materiales locales, que se extraen y fabrican dentro de la región, reduciendo los impactos ambientales que surgen del transporte; BREEAM promueve el uso de materiales de bajo impacto ambiental durante el ciclo de vida del edificio; y VERDE propone reducir los impactos asociados a la producción de los materiales de construcción.
En la evaluación del rendimiento ambiental del edificio, en su proceso constructivo, los materiales cumplen un rol importantísimo, pues sus características e impactos en el medio ambiente permiten evaluar el edificio.

"Sin embargo, publicaciones científicas han expuesto las dificultades que estos métodos tienen para aplicarse en regiones diferentes para las cuales fueron desarrollados" (Quesada, Calle, Guillén, Ortiz y Lema, 2018, p. 207), y en países en vías de desarrollo esta situación puede agudizarse, por esto la importancia de desarrollar métodos de evaluación nacionales y locales.

En este contexto su investigación desarrolló: - un Método de Evaluación Sustentable (BSA) para viviendas de la ciudad de Cuenca, y - una herramienta: "Certificación edificio Sustentable y Seguro (CESSuc)", para medir el desempeño de las edificaciones, basado en indicadores apropiados para la localidad.

\section{Contexto nacional y local}

\section{Normativa ambiental ecuatoriana}

Según el Primer Informe Bienal de Actualización del Ecuador, este fue el primer país en el mundo al reconocer los Derechos de la Naturaleza a través de su Constitución en 2008, resaltando su compromiso con la conservación y la gestión ambiental. Así se garantiza que se respete integralmente su existencia.

Igualmente, el Ecuador suscribió la Convención Marco de las Naciones Unidas sobre el Cambio Climático (CMNUCC) en el año 1994; ratificó el Protocolo de Kioto ${ }^{1}$ en 1999; firmó el Acuerdo de París

\footnotetext{
${ }^{4}$ El protocolo de Kioto se adoptó en Japón, el 11 de diciembre de 1.997 y fue firmado por 84 países. Las partes incluidas en su anexo I se comprometen a lograr objetivos individuales y jurídicamente vinculantes para limitar o reducir sus emisiones de gases de efecto invernadero. Sólo los países que lo ratifiquen estarán obligados a su cumplimiento. Se puso en marcha en 2.005 gracias a la ratificación de un número de países equivalente al $55 \%$ de la generación de $\mathrm{CO} 2$ del mundo. Su objetivo es reducir las emisiones de seis gases relacionados con el calentamiento de la atmósfera a partir del efecto invernadero y detener el cambio climático que aumentará en este siglo entre 1,4 y $5,8^{\circ} \mathrm{C}$ la temperatura de la superficie de la Tierra.
} 
en 2016 y ha participado activamente del proceso de negociaciones internacionales de cambio climático, al tiempo que ha generado un marco regulatorio e institucional que permite fortalecer el cumplimiento de los objetivos establecidos en la Convención.
Es importante mencionar que Ecuador, al ser un país en desarrollo, o No-Anexo I, no está obligado a reducir emisiones de gases de efecto invernadero (GEI). Sin embargo, está convencido de que su propio desarrollo debe estar vinculado a las prioridades del cambio climático, lo cual se concreta a través de la creación de una gobernanza basada en la cosmovisión de los pueblos ancestrales, el Sumak Kawsay² o Buen Vivir (Ministerio del Ambiente, 2016, p. 4).

Figura 2. Total de emisiones netas de Co2

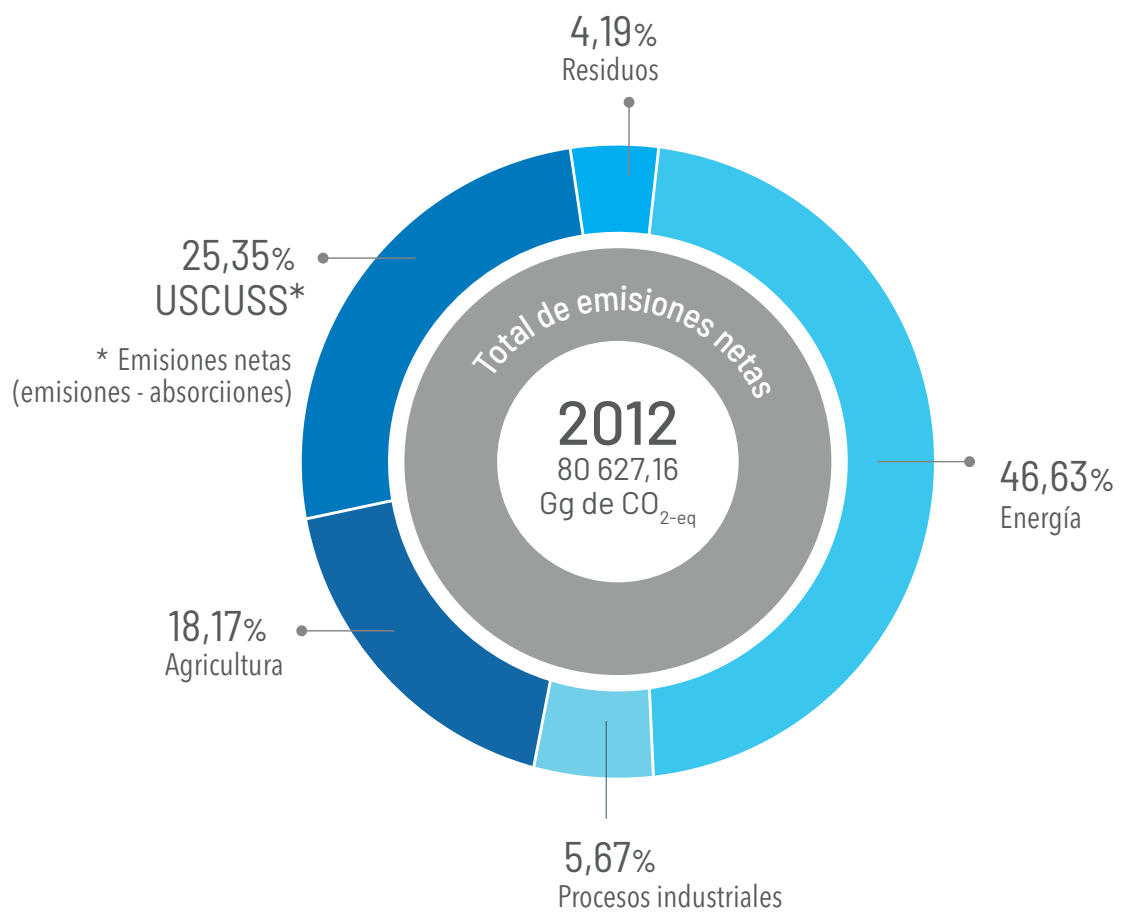

De Ministerio del Ambiente, MAE, (2016).

${ }^{5}$ El Sumak Kawsay, como forma de vida en armonía con la naturaleza, no es simplemente una forma de economía sostenible, sino que tiene un sentido más transcendente que enlaza con las creencias de los pueblos indígenas. Los pueblos indígenas entienden la naturaleza, con una perspectiva holística, como un ente vivo que lo engloba todo, incluidos los seres humanos. Para el indígena su destino es cuidar a la naturaleza como un ente del que forma parte; por eso, no debe tomarse de ella más que lo necesario para la subsistencia, pidiendo permiso mediante rituales (como los de la siembra, los de la cosecha o los de la caza) y dándole las gracias por sus dones mediante las ofrendas (tumines). 
Ecuador apuesta por un desarrollo sustentable en todos los ámbitos de la vida de una Nación. Pero, a pesar de todos estos esfuerzos, en el ámbito referido a las prácticas de construcción sustentable, al igual que otros países de Latinoamérica, apenas ha empezado a incursionar y esto debido a la falta de normativas y regulaciones que ayuden al cumplimiento de estos parámetros. "Motivo por lo cual se sigue construyendo edificios en forma convencional y generando impactos ambientales" (Daza, 2010, p. 2). En Ecuador cerca de $9 \%$ del producto interno bruto (PIB), un valor significativo, corresponde a la construcción (Daza, 2010).

\section{Norma Ecuatoriana de la Construcción}

La Norma Ecuatoriana de Construcción (NEC) busca la calidad de vida de los ecuatorianos y se constituye en normativa que aporta principios básicos para el diseño de estructuras; estableciendo parámetros mínimos de seguridad y calidad en las edificaciones; optimizando los mecanismos de control y mantenimiento en los procesos constructivos; reduciendo el consumo y mejorando la eficiencia energética de las edificaciones. En definitiva, respalda el cumplimiento de los principios básicos de habitabilidad y salud y fija responsabilidades, obligaciones y derechos de todos los actores involucrados en la construcción. La NEC es de cumplimiento obligatorio a nivel nacional y debe ser considerada en todos los procesos constructivos.

En la actualidad, en el país existen varios profesionales de la construcción que buscan llevar a cabo proyectos que sean sustentables, y ante esta realidad la NEC, en su capítulo 13 de Eficiencia Energética en la Construcción en Ecuador, fomenta el diseño y construcción de edificaciones bajo puntos de vista de sostenibilidad, eficiencia y buen manejo de los recursos, disminuyendo, de esta manera, el consumo de combustibles fósiles y recursos no renovables y las emisiones de gases de efecto invernadero asociadas.
Pero a causa de la falta, por muchos años, de regulaciones o normas técnicas sustentables en nuestro país, los proyectos de arquitectura y diseño en la ciudad de Cuenca, como en muchas de las ciudades del Ecuador, se han ejecutado sin un sustento medioambiental, trayendo como consecuencia construcciones poco eficientes y daños irreparables al medio ambiente (Serrano et al., 2015).

\section{Materiales utilizados en la construcción nacional}

El consumo energético derivado de las actividades de construcción se da, por un lado, en el proceso específico de la obra y, por otro, en la manufactura de los materiales. "Materiales como acero estructural, cemento, hormigón armado y aluminio, consumen grandes cantidades de energía durante su producción, con importantes emisiones de $\mathrm{CO}_{2}$ derivadas de los procesos de manufactura" (Pelufo, 2011, p. 44).

Según la información disponible del Instituto Nacional de Estadística y Censos (INEC), en la Encuesta de Edificaciones (2018), el hormigón es el material más usado para construir en Ecuador. Los cimientos y la estructura de hormigón predominan en las construcciones que se hacen cada año en el país y su preferencia responde a la durabilidad. 
Figura 3. Materiales predominantes a ser utilizados en la edificación

MATERIALES PREDOMINANTES A SER UTILIZADOS EN LA EDIFICACIÓN

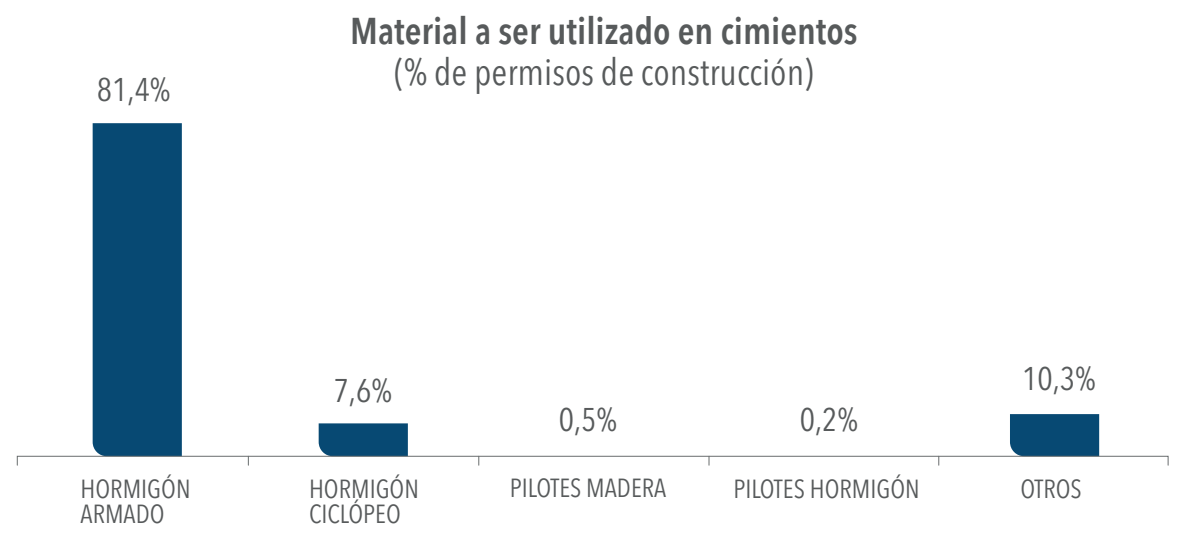

\section{Material a ser utilizado en estructura}

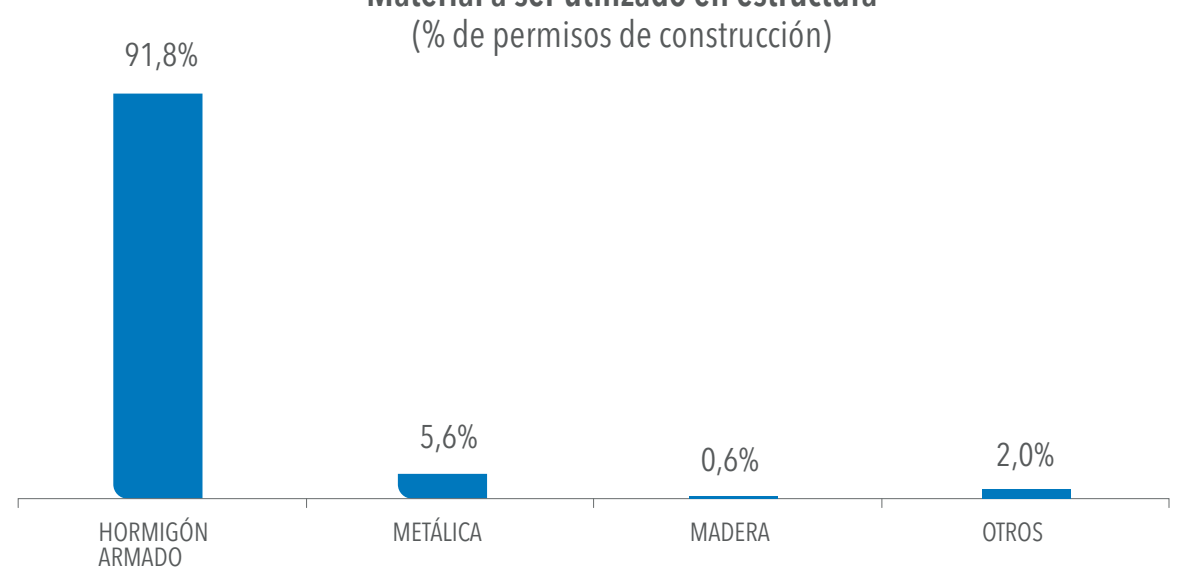

Material a ser utilizado en cubierta

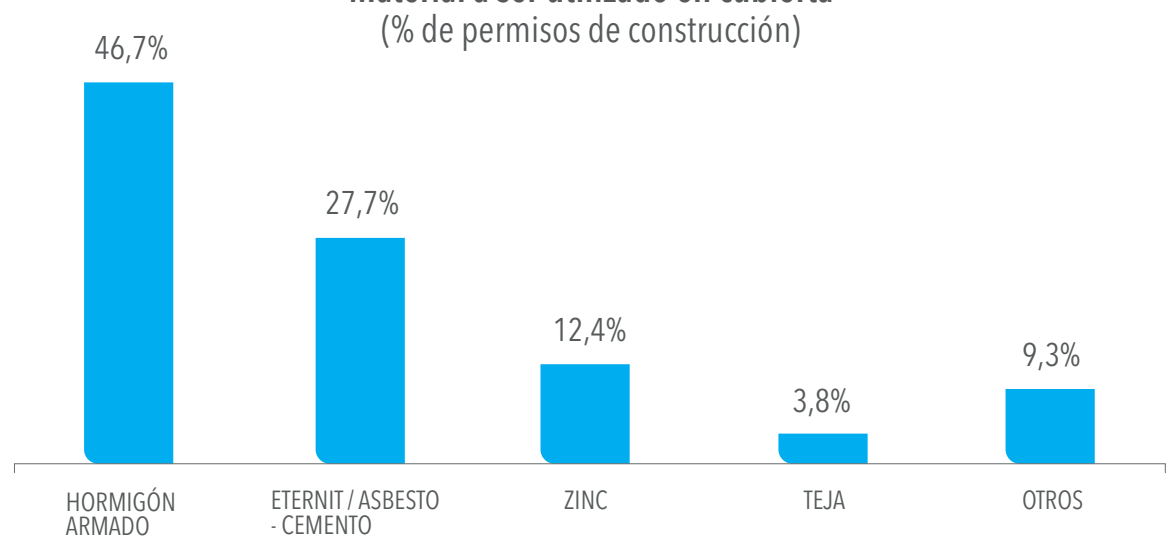




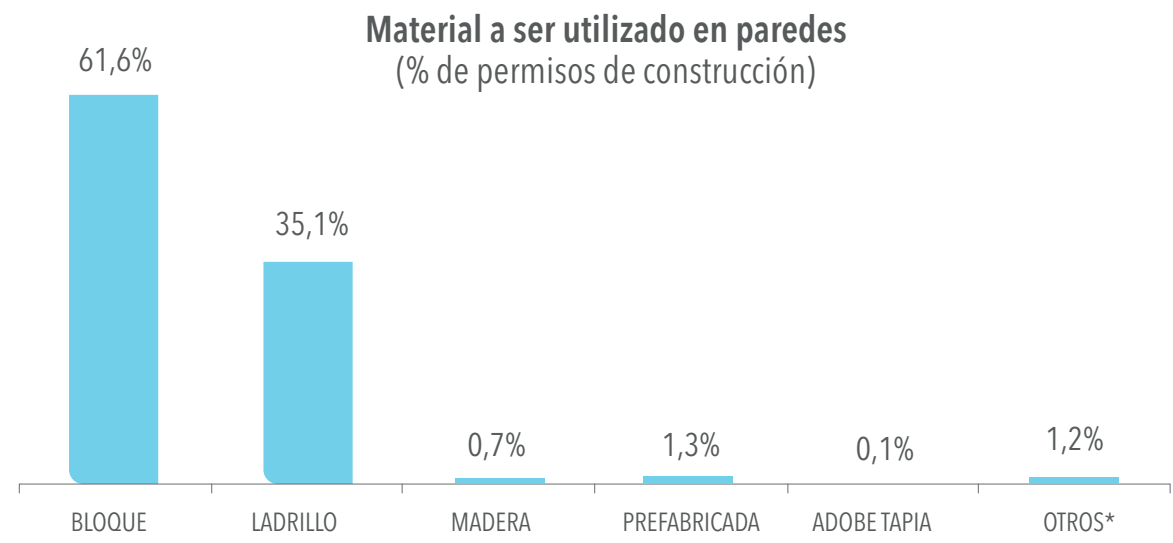

* La categoría Otros en paredes también incluye Caña revestida o bareque

De Instituto Nacional de Estadísticas y Censos. Encuesta de Edificaciones, (2016).

\section{Materiales utilizados en la construcción local}

Cuenca posee gran cantidad de edificaciones construidas con adobe. "Sin embargo, a partir de la llegada del movimiento moderno a la ciudad y el incremento en la utilización del cemento y otros materiales "modernos" en la construcción, se generó una ruptura en el proceso de construcción tradicional" (Orellana, 2017, p. 2).

Es el Centro Histórico de la ciudad en donde se emplaza la mayor cantidad de edificaciones construidas con adobe, de acuerdo al inventario realizado por el $\mathrm{GAD}^{3}$ Municipal. La importancia que tuvo este sistema constructivo se ve reflejado en los muros de adobe, que son elementos comunes en los edificios patrimoniales. Pero el uso de la tierra, poco a poco, fue sustituido por los materiales industrializados como el concreto, vidrio y acero, que la modernidad trajo consigo.

\section{Metodología}

\section{Antecedentes y homólogos}

La actividad constructiva utiliza recursos que se obtienen de la naturaleza y, para esto, se requiere de enormes cantidades de energía, tanto para la explotación como para su conversión en productos de construcción; todos los desechos y emanaciones de esta acción son depositados en el ambiente, durante y final del ciclo de vida de los productos y obras, con las consiguientes emisiones a la atmósfera (Mercader, Ramírez de Arellano y Olivares, 2012).

Contribuir con la sostenibilidad "demanda interés para identificar y cuantificar los factores que condicionan el impacto ambiental, pues la única forma para hablar de mejoras es obteniendo datos que se puedan medir y contrastar con facilidad" (Mercader, Ramírez de Arellano y Olivares, 2010, p. 125).

${ }^{3}$ Gobiernos Autónomos Descentralizados. 
Dentro del Diseño de Interiores existe poco sustento teórico concerniente al diseño interior sustentable y este es uno de los impulsos para el desarrollo de esta investigación, tomando referencias de autores que tratan temas relacionados a la construcción, arquitectura y materiales.

En las propuestas de diseño interior los materiales constructivos desempeñan un rol muy importante, ya que su aplicación dota a los espacios de carácter. "En interiorismo se relacionan los acabados que se aplican en las superficies, tanto en pisos, paredes y cielo raso y al mismo tiempo la materia prima que constituye el mobiliario 0 accesorios propuestos" (Corral, 2017, s.n). De igual manera, al momento de diseñar es muy importante cuantificar el material a colocar para evitar sobrantes.

Para conocer el impacto ambiental que producen, el modelo de evaluación pretende identificar y cuantificar los materiales utilizados en intervenciones de remodelación interior de espacios comerciales, restaurantes y cafeterías, considerando el consumo energético y las emisiones de $\mathrm{CO}_{2}$ desde la óptica de la fabricación de los mismos, no incluyendo transportes asociados, ni puesta en obra, siendo esta una herramienta que permita tomar decisiones más acertadas al momento de seleccionar materiales. Para esto se tomó como referencia el modelo metodológico utilizado en el trabajo de investigación denominado "Cuantificación de los recursos consumidos y emisiones de $\mathrm{CO}_{2}$ producidas en las construcciones de Andalucía y sus implicaciones en el protocolo de Kioto (2010)", realizada por Mercader, Ramírez de Arellano y Olivares, y la base de datos de materiales de construcción del Banco Estructurado de elementos Constructivos (BEDEC) del Instituto de Tecnología de la Construcción de Cataluña (ITeC), que es la referencia más próxima a los materiales de construcción empleados en las construcciones de nuestro país. Pero, debido a que esta base de datos no contiene todos los materiales necesarios a ser evaluados se utilizó también la base de datos Inventory of Carbon \& Energy (ICE), desarrollada por la University of
Bath, del Reino Unido, que al ser comparada con los valores de la base de datos española se obtuvieron indicadores similares entre sí.

Es importante considerar que los datos referidos a la energía consumida por un determinado material en su fabricación y las emisiones de $\mathrm{CO}_{2}$, derivadas del proceso, son referenciales y puede haber variaciones considerables en función al ámbito de aplicación internacional.

Esta investigación limita el estudio de espacios comerciales, restaurantes y cafeterías, pues la sustentabilidad engloba todos los elementos constituyentes del negocio, incluyendo productos y servicios que ofrece. Los restaurantes y cafeterías se caracterizan por pensar en un desarrollo más amigable con el medioambiente, utilizando por lo general productos locales, orgánicos en su mayoría y biodegradables.

Si bien la mentalidad de los consumidores ha cambiado, al ser cada vez más aquellos que tienen interiorizado el respeto al entorno y sustentabilidad y en base a ello toman decisiones diarias con las marcas, en nuestro país las empresas llevan retraso en la aplicación de iniciativas "verdes" o ecológicas, en comparación con países desarrollados.

Como diseñadores de espacios interiores es evidente que los materiales que utilizamos en las intervenciones son, también, responsables del daño medio ambiental, debido al gran consumo de energía y emisiones en su proceso de fabricación. El reconocimiento de estos aspectos técnicos en la configuración de propuestas responsables es uno de los pilares fundamentales de esta investigación; misma que pretende identificar cuáles son los materiales habituales utilizados por profesionales, del medio local, en las intervenciones comerciales, restaurantes y cafeterías; para, de esta manera, generar una base de datos que nos permita recopilar información sobre indicadores medioambientales, en relación a otros países, para evaluar los impactos del consumo energético y emisiones de dióxido de carbono $\left(\mathrm{CO}_{2}\right)$ en su proceso de fabricación. 
En este trabajo no se analizará la iluminación de los proyectos con la finalidad de acotar el estudio y garantizar la fiabilidad de los resultados, sin embargo es un punto muy importante a considerar en la etapa de Diseño. La iluminación eficiente debería ser el objetivo de cualquier proyecto, que permita reducir los impactos ambientales y conseguir espacios confortables. Elegir luminarias es sólo el primer paso para crear un sistema de iluminación eficiente, además existen otros criterios que deben ser considerados, como la correcta colocación de las luminarias o la cantidad de las mismas. Es importante considerar que una correcta iluminación ayuda a los clientes a disfrutar de una verdadera experiencia, y el aprovechamiento de la luz natural debe ser parte fundamental de los proyectos para reducir los impactos ambientales.

El modelo de análisis propuesto permite autoevaluar, de forma estimativa, las emisiones de $\mathrm{CO}_{2}$ y consumo energético de los materiales derivados de su proceso de fabricación y que son utilizados en las intervenciones de espacios interiores comerciales, en restaurantes y cafeterías, para, de esta manera, tomar decisiones acertadas en la generación de espacios óptimos, que tengan un mínimo de impacto ambiental.
Se establecen cuatro niveles ordenados jerárquicamente que, a su vez, se dividen en etapas y subetapas, que atenderán al mismo orden, dando cumplimiento al objetivo principal de la investigación: desarrollar un modelo de evaluación para aplicarlo en tres intervenciones de remodelación interior comercial, de restaurantes y cafeterías, con materiales vernáculos, modernos y contemporáneos; cuantificando el consumo energético ${ }^{4}$ y emisiones de $\mathrm{CO}_{2}{ }_{2}$, derivados del proceso de fabricación de los mismos $y$, de esta manera, determinar el grado de impacto ambiental, para, a partir de aquí, generar propuestas más sustentables.

La siguiente figura ilustra esta estructura jerárquica:

${ }^{4}$ Consumo energético de los materiales de construcción, entendida como la cantidad de energía consumida o que se estime necesaria para satisfacer distintas necesidades asociadas a su proceso productivo. Dicha magnitud queda reflejada en un indicador cuantitativo expresado en $\mathrm{MJ} / \mathrm{kg}$ de material de construcción.

${ }^{5}$ Emisiones de $\mathrm{CO}_{2}$ de los materiales de construcción, la cantidad de emisiones de gas carbónico $\left(\mathrm{CO}_{2}\right)$, derivadas del proceso productivo de los materiales de construcción. Dicha magnitud queda reflejada en un indicador cuantitativo expresado en $\mathrm{kgCO}_{2} /$ $\mathrm{kg}$ de material de construcción. 
Figura 4. Esquema metodológico

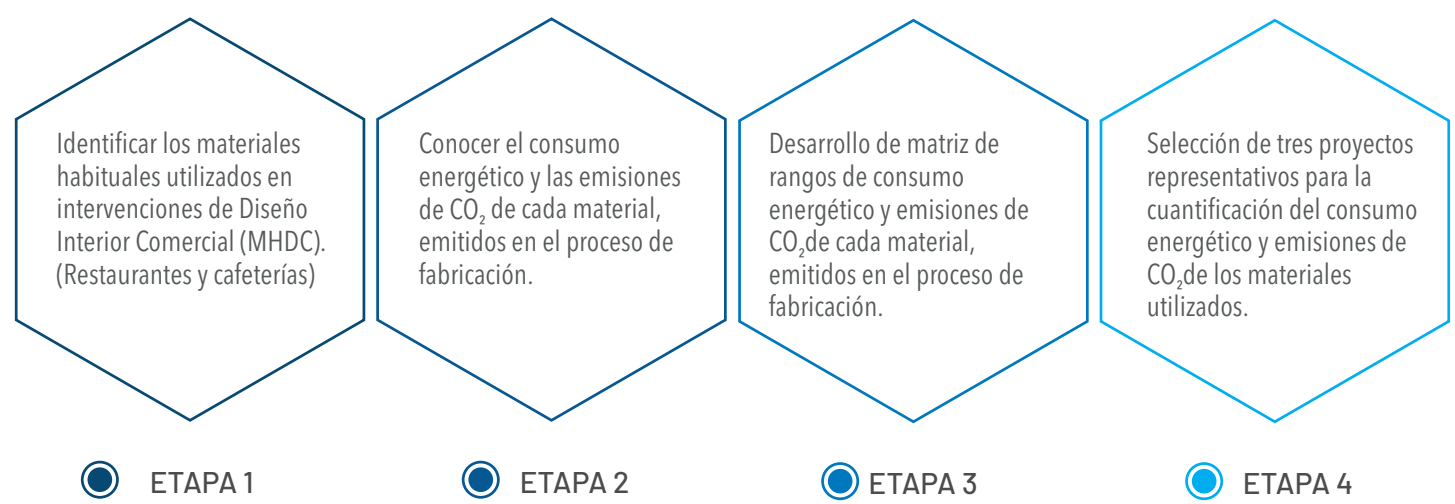

E1.1: Base de datos obtenida de profesionales de Diseño Interior del medio.

E1.2: Determinar los materiales más habituales utilizados en las diferentes bases de datos.

E1.3: Elección de la muestra de los materiales (MHDC).
E2.1: Obtención de base de datos con información medioambiental, de referencia.

E2.2: Consumo energético por material.

E2.3: Emisiones de $\mathrm{CO}_{2}$ por material
E3.1: Determinar rangos de impacto medioambiental por consumo energético y emisiones de $\mathrm{CO}_{2}$ de cada material
E4.1: Cuantificación de los materiales utilizados en la ejecución de los proyectos.

E4.2: Homogenización de medidas.

E4.3: Convención en cantidades por $\mathrm{m}_{2}$ construido.

E4.4: Normalización

E4.5: Determinación de componentes representativos de la muestra.

E4.6: Determinación de consumo energético y emisiones de $\mathrm{CO}_{2}$ de los materiales.

E4.7: Consumo por $m_{2}$ construido.
Para conseguir el objetivo expuesto en el nivel 1: identificar los materiales habituales utilizados en intervenciones de Diseño Interior Comercial (MHDC), en restaurantes y cafeterías, es necesario llevar a cabo la etapa 1, subdividida a su vez en subetapas:

E1.1. Consiste en recopilar información de los materiales utilizados en intervenciones de diseño interior en restaurantes y cafeterías, realizados por diferentes profesionales del medio local (Base de datos).
E1.2. Determinar los materiales que más se repiten en los diferentes proyectos.

E 1.3. Seleccionar la muestra de los materiales (MHDC). 
Tabla 1. Materiales Habituales de intervenciones de Diseño Interior Comercial en restaurantes y cafeterías (MHDC)

\begin{tabular}{|l|l|}
\hline Acero galvanizado & Mortero prefabricado \\
\hline Acero negro & Papel tapiz papel \\
\hline Adhesivo de poliuretano & Pintura látex \\
\hline Adhesivo de PVC & Pintura plástica \\
\hline Aluminio & Piso de vinil \\
\hline Barniz & Poliéster \\
\hline Cemento Pulido hormigón prefabricado & Polipropileno \\
\hline Cerámica / Gres esmaltado & Poliuretano/ Esponja \\
\hline Cola natural & Polvo de cuarzo \\
\hline Disolvente & Resina expóxica \\
\hline Empaste / mortero pref. silicio - calcáreo & Silicona \\
\hline Empore / Adhesivo de resinas epoxicas & Tablero de partículas de madera \\
\hline Enlucido / mortero prefabricado & Tablero aglomerado \\
\hline Esmalte de poliuretano & Tablero de partículas de madera chapada \\
\hline Fachaleta de ladrillo / Arcilla cocida & Vidrio \\
\hline Fibra natural & Vidrio templado \\
\hline Granito / piedra natural & Vinil adhesivo / Vinilo \\
\hline Gres porcelánico & Yeso cartón \\
\hline Madera natural & Yeso \\
\hline Masilla acrílica & PVC \\
\hline Masilla de poliuretano & PVC tubería \\
\hline Metacrilato & \\
\hline
\end{tabular}

Para conseguir el objetivo expuesto en el nivel 2: conocer el consumo energético y emisiones de $\mathrm{CO}_{2}$ de cada material emitido en el proceso de fabricación, es necesario llevar a cabo la etapa 2, subdividida a su vez en las subetapas:

E2.1. Obtención de base de datos con información medioambiental, la base de datos de referencia utilizada será la española BEDEC (Instituto de Tecnología de la Construcción de Cataluña) donde cada material es catalogado del análisis de ciclo de vida, es decir desde la materia prima hasta la producción del material de construcción, facilitada por miembros del ITeC, que recoge el consumo ener- gético producido en la fabricación de los materiales de construcción citados y las emisiones de $\mathrm{CO}_{2}$ derivadas del proceso hasta la puerta de la fábrica, no incluye ni transportes asociados ni puesta en obra. Debido a que la base de datos española no contiene todos los materiales necesarios a ser evaluados se ha utilizado, también, la base de datos Inventory of Carbon \& Energy (ICE), desarrollada por la University of Bath, UK (2008).

E2.2. Determinación del consumo energético de cada material.

E2.3. Determinación de las emisiones de $\mathrm{CO}_{2}$ de cada material constitutivo del listado (MHDC). 
Tabla 2. Base de Datos Ambientales (MHDC)

\begin{tabular}{|c|c|c|}
\hline Acero galvanizado & 39.40 & 3.91451 \\
\hline Acero negro & 24.40 & 1.95000 \\
\hline Adhesivo de poliuretano & 45.00 & 6.64200 \\
\hline Adhesivo de PVC & 45.00 & 6.64200 \\
\hline Aluminio & 205.00 & 30.13977 \\
\hline Barniz & 100.00 & 14.76000 \\
\hline Cemento Pulido hormigón prefabricado & 4.80 & 0.456000 \\
\hline Cerámica / Gres esmaltado & 10.90 & 0.81750 \\
\hline Cola natural & 5.00 & 0.45000 \\
\hline Disolvente & 100.00 & 14.76000 \\
\hline Empaste / mortero pref. silicio - calcáreo & 2.00 & 0.19000 \\
\hline Empore / Adhesivo de resinas epoxicas & 45.00 & 6.64200 \\
\hline Enlucido / mortero prefabricado & 2.35 & 0.22325 \\
\hline Esmalte de poliuretano & 70.00 & 10.33000 \\
\hline Fachaleta de ladrillo / Arcilla cocida & 8.20 & 0.52000 \\
\hline Fibra natural & 1.70 & 0.15300 \\
\hline Granito / piedra natural & 0.18 & 0.01800 \\
\hline Gres porcelánico & 10.90 & 1.02460 \\
\hline Madera natural & 2.100 & 0.06300 \\
\hline Masilla acrílica & 20.000 & 2.95000 \\
\hline Masilla de poliuretano & 20.000 & 2.95000 \\
\hline Metacrilato & 53.720 & 7.92907 \\
\hline Mortero prefabricado & 2.350 & 0.22325 \\
\hline Papel tapiz papel & 31.10 & 1.803800 \\
\hline Pintura látex & 24.70 & 3.64325 \\
\hline Pintura plástica & 20.00 & 2.95000 \\
\hline Piso de vinil & 65.64 & 2.29000 \\
\hline Poliéster & 53.72 & 7.92907 \\
\hline Polipropileno & 79.00 & 11.66040 \\
\hline Poliuretano/ Esponja & 72.10 & 3.00000 \\
\hline Polvo de cuarzo & 7.00 & 0.63000 \\
\hline Resina expóxica & 93.00 & 13.72680 \\
\hline Silicona & 113.00 & 16.67880 \\
\hline Tablero de partículas de madera & 15.000 & 1.35000 \\
\hline Tablero aglomerado & 25.80 & 1.52957 \\
\hline Tablero de partículas de madera chapada & 18.90 & 1.70100 \\
\hline Vidrio & 15.90 & 0.93726 \\
\hline Vidrio templado & 26.20 & 1.544420 \\
\hline Vinil adhesivo / Vinilo & 80.00 & 11.81000 \\
\hline Yeso cartón & 7.90 & 0.47400 \\
\hline Yeso & 2.57 & 0.23987 \\
\hline PVC & 70.00 & 10.33375 \\
\hline \multirow[t]{2}{*}{ PVC tubería } & 67.50 & 2.50000 \\
\hline & $\mathrm{MJ} / \mathrm{Kg}$ & $\mathrm{Kg} \mathrm{CO}_{2} / \mathrm{Kg}$ \\
\hline
\end{tabular}


En el nivel 3 el objetivo a cumplir es: desarrollar la matriz de rangos de consumo energético y emisiones de $\mathrm{CO}_{2}$ de cada material, emitidos en el proceso de fabricación y que forman parte del listado de los materiales habituales más utilizados en intervenciones de Diseño Interior Comercial. En esta etapa 3, el camino a seguir está en la subetapa 3.1.
E3.1. Determinación de rangos de impacto medioambiental por consumo energético y emisiones de $\mathrm{CO}_{2}$ de cada material, obtenidos de la base de datos, organizándola de mayor a menor y diferenciándola por colores del más alto al más bajo.

Tabla 3. Matriz de rangos de Impacto Ambiental (MHDC)

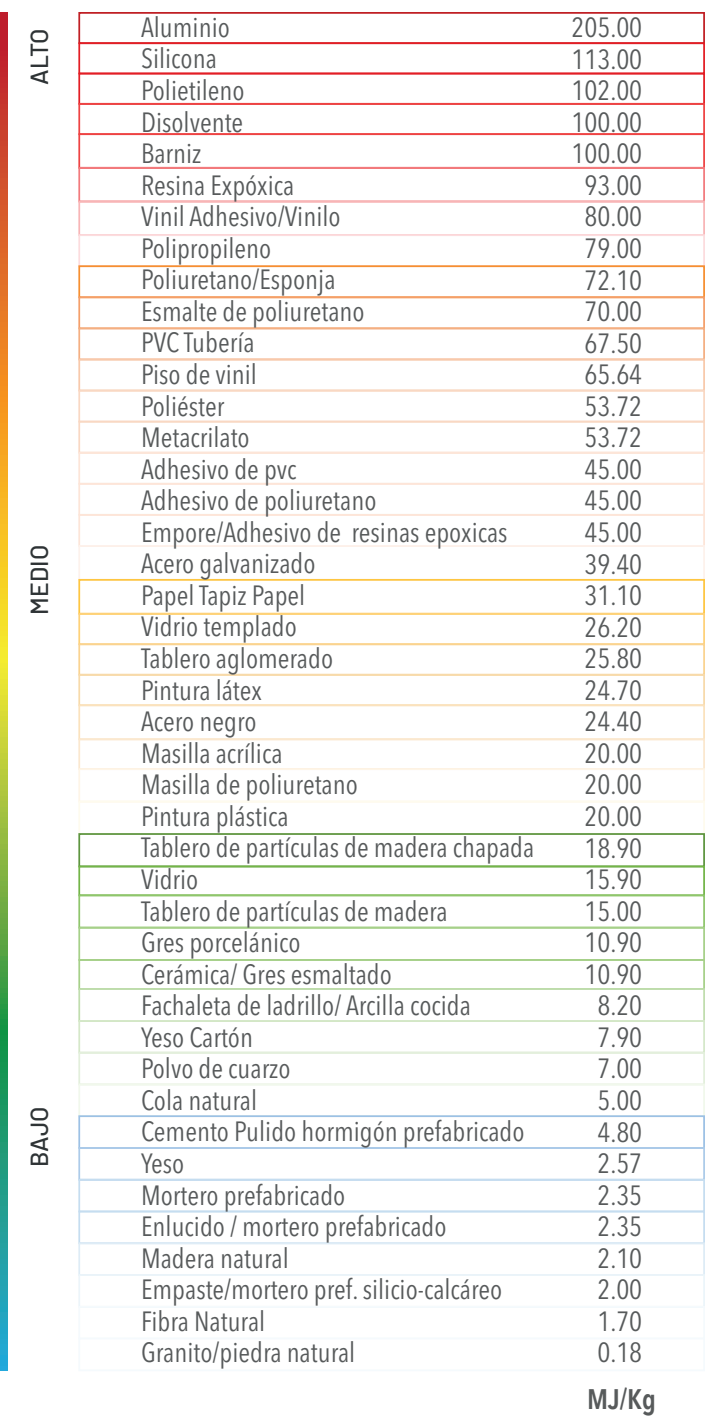

\begin{tabular}{|l|l|}
\hline Aluminio & 30.13977 \\
\hline Silicona & 16.67880 \\
\hline Polietileno & 15.06156 \\
\hline Disolvente & 14.76000 \\
\hline Barniz & 14.76000 \\
\hline Resina Expóxica & 13.72680 \\
\hline Vinil Adhesivo/Vinilo & 11.81000 \\
\hline Polipropileno & 11.66040 \\
\hline Esmalte de poliuretano & 10.33000 \\
\hline Metacrilato & 7.92907 \\
\hline Poliéster & 7.92907 \\
\hline Adhesivo de poliuretano & 6.64200 \\
\hline Adhesivo de pvc & 6.64200 \\
\hline Empore/Adhesivo de resinas epoxicas & 6.64200 \\
\hline Acero galvanizado & 3.91451 \\
\hline Pintura látex & 3.64325 \\
\hline Poliuretano/Esponja & 3.00000 \\
\hline Masilla acrílica & 2.95000 \\
\hline Masilla de poliuretano & 2.95000 \\
\hline Pintura plástica & 2.95000 \\
\hline PVCTubería & 2.50000 \\
\hline Piso de vinil & 2.29000 \\
\hline Acero negro & 1.95000 \\
\hline Papel Tapiz Papel & 1.80380 \\
\hline Tablero de partículas de madera chapada & 1.70100 \\
\hline Vidrio templado & 1.54442 \\
\hline Tablero aglomerado & 1.52957 \\
\hline Tablero de partículas de madera & 1.35000 \\
\hline Gres porcelánico & 1.02460 \\
\hline Vidrio & 0.93726 \\
\hline Cerámica/ Gres esmaltado & 0.81750 \\
\hline Polvo de cuarzo & 0.63000 \\
\hline Fachaleta de ladrillo/ Arcilla cocida & 0.52000 \\
\hline Yeso Cartón & 0.47400 \\
\hline Cemento Pulido hormigón prefabricado & 0.45600 \\
\hline Cola natural & 0.45000 \\
\hline Yeso & 0.23987 \\
\hline Mortero prefabricado & 0.22325 \\
\hline Enlucido / mortero prefabricado & 0.22325 \\
\hline Empaste/mortero pref. silicio-calcáreo & 0.19000 \\
\hline Fibra Natural & 0.15300 \\
\hline Granito/piedra natural & 0.06300 \\
\hline
\end{tabular}

$\mathrm{KgCO} 2 / \mathrm{Kg}$ 
En el nivel de la etapa 4 se selecciona los tres proyectos para la cuantificación del consumo energético y emisiones de $\mathrm{CO}_{2}$ de los materiales utilizados, subdividida a su vez en las subetapas:

E4.1. Cuantificación de los materiales utilizados en cada proyecto, materiales consumidos en su ejecución. El documento que proporciona la me- jor información para cuantificar los materiales de un proyecto es el presupuesto de la obra. Este documento, que pertenece al proyecto de ejecución, recoge un listado de los diferentes materiales, cuantificados en unidades de medida que mejor se ajustan a su forma y uso al que se destinan en la obra.

Tabla 4. Cuantificación de los materiales utilizados en cada proyecto

\section{PRESUPUESTO FABRICACIÓN DE MOBILIARIO PARA CAFÉ EL MERCADO}

\begin{tabular}{|c|c|c|c|c|}
\hline DESCRIPCIÓN & UND & CANT. & P. UNIT & TOTAL \\
\hline \multicolumn{5}{|l|}{ PISO } \\
\hline Porcelanato & $m^{2}$ & 0 & & - \\
\hline \multicolumn{5}{|l|}{ PAREDES } \\
\hline $\begin{array}{l}\text { Pintura interior } \\
\text { Fachaleta de ladrillo }\end{array}$ & $\begin{array}{l}\mathrm{m}^{2} \\
\mathrm{~m}^{2}\end{array}$ & $\begin{array}{l}29.63 \\
8.05\end{array}$ & & - \\
\hline \multicolumn{5}{|l|}{ CIELO RASO } \\
\hline Estuco & $m^{2}$ & 0 & & - \\
\hline \multicolumn{5}{|l|}{ MOBILIARIO } \\
\hline Couter según diseño en melanina y mdf lacado & UND & 1 & & - \\
\hline Forro para frigorífico en mdf lacado & UND & 1 & & - \\
\hline Estantes metálicos con repisas de melanina & UND & 1 & & - \\
\hline Estantes metálicos con repisas de mdf lacado & UND & 2 & & - \\
\hline Marco de madera para letrero & UND & 1 & & - \\
\hline Estante 1 para café en estructura metálica y repisas de madera & UND & 1 & & - \\
\hline Estante 2 para café en estructura metálica y repisa de madera & UND & 1 & & - \\
\hline Estante para plantas en estructura metálica y repisa e madera & UND & 1 & & \\
\hline Mueble de trabajo en melanina y mesón de cuarzo & UND & 1 & & - \\
\hline Sillas según modelo en madera & UND & 8 & & - \\
\hline Mesas según modelo en madera y metal & UND & 2 & & - \\
\hline
\end{tabular}

E4.2. Homogeneización de medidas.

En esta subetapa, los datos correspondientes a los materiales de los proyectos de ejecución se- leccionados se adecuaron, en base a una estructura común, para facilitar su análisis. Obteniendo, así, un listado de cantidades y unidades de medida original. 
Tabla 5. Homogeneización de medidas

\begin{tabular}{|c|c|c|}
\hline $\begin{array}{l}\text { PRESUPUESTO FABRICACION DE } \\
\text { MOBILIARIO PARA CAFÉ EL MERCADO }\end{array}$ & UND & CANT. \\
\hline \multicolumn{3}{|l|}{ PISO } \\
\hline Porcelanato/Gres porcelanato & $\mathrm{m}^{2}$ & 0 \\
\hline \multicolumn{3}{|l|}{ PAREDES } \\
\hline $\begin{array}{l}\text { Pintura látex } \\
\text { Fachaleta de ladrillo }\end{array}$ & $\begin{array}{l}\mathrm{m}^{2} \\
\mathrm{~m}^{2}\end{array}$ & $\begin{array}{l}37.68 \\
8.05\end{array}$ \\
\hline \multicolumn{3}{|l|}{ CIELO RASO } \\
\hline Estuco/Yeso & $\mathrm{m}^{2}$ & 0 \\
\hline \multicolumn{3}{|l|}{ MOBILIARIO } \\
\hline Madera natural & $\mathrm{m}^{2}$ & 12.81 \\
\hline Tablero de partículas de madera & $\mathrm{m}^{2}$ & 4.76 \\
\hline Tablero de partículas de madera chapada & $\mathrm{m}^{2}$ & 16.65 \\
\hline Acero negro & $\mathrm{ml}$ & 84 \\
\hline
\end{tabular}

E4.3. Conversión en cantidades por $\mathrm{m}^{2}$ construido.

Para obtener el valor que representa cada material, respecto al área total construida de los proyectos, se dividió las cantidades correspondientes a los materiales empleados en la ejecución sobre la superficie construida de cada uno de los proyectos. Obteniendo así, para cada material, las cantidades originales por $\mathrm{m}^{2}$ de superficie construida. 
Tabla 6. Conversión en cantidades por $\mathrm{m}^{2}$ construido

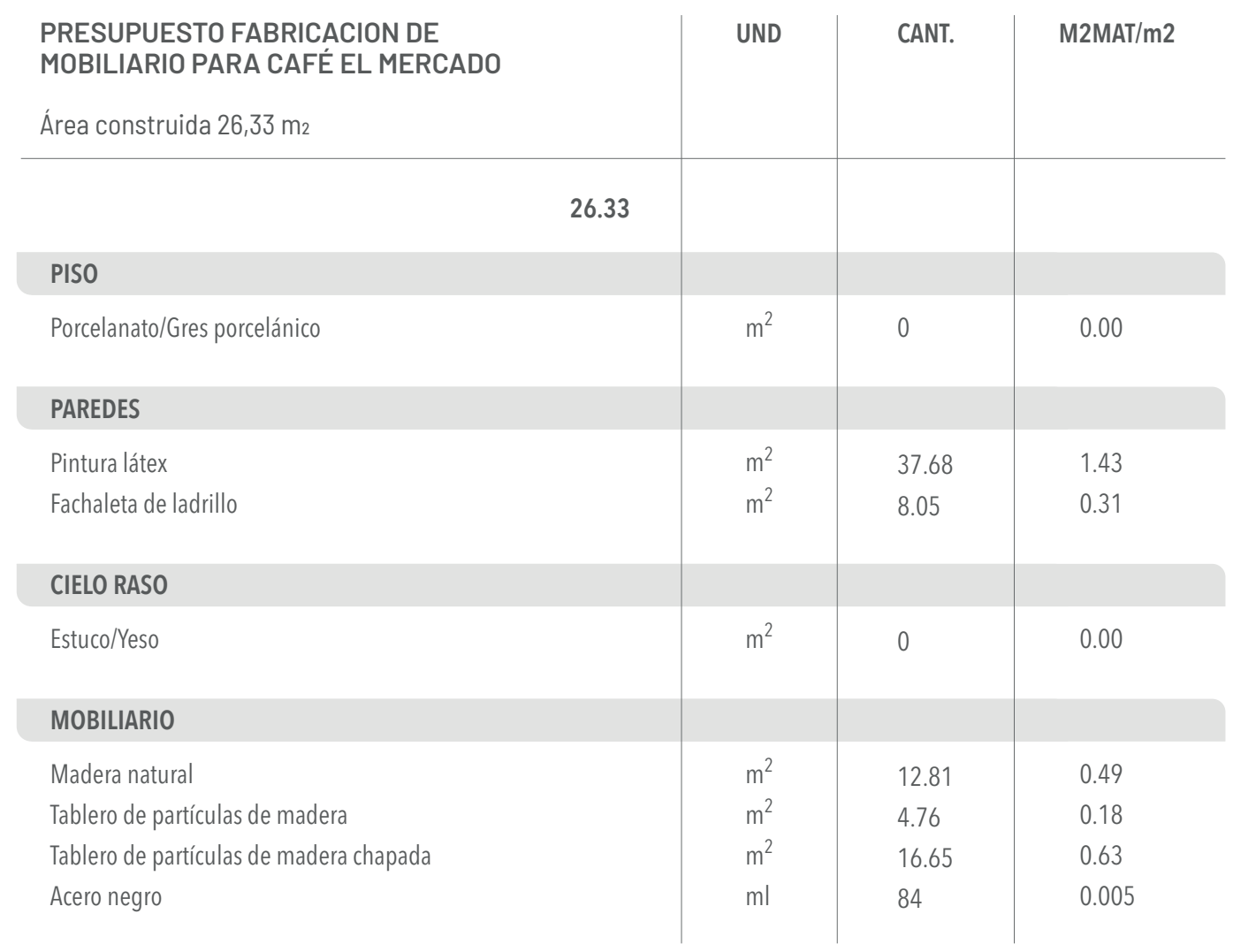

E4.4. Normalización de medidas.

Es necesario expresar los resultados obtenidos en la subetapa anterior en una misma unidad de medida. La normalización de las unidades resultantes consiste en transformar la unidad origen, unidad de medida del elemento, a la unidad de destino, el peso en kg del elemento, utilizando información comercial de los productos a través de catálogos 0 internet; siendo aproximaciones referenciales, dado que los valores correspondientes al peso del elemento pueden variar en función de la marca comercial. 
Tabla 7. Normalización de medidas

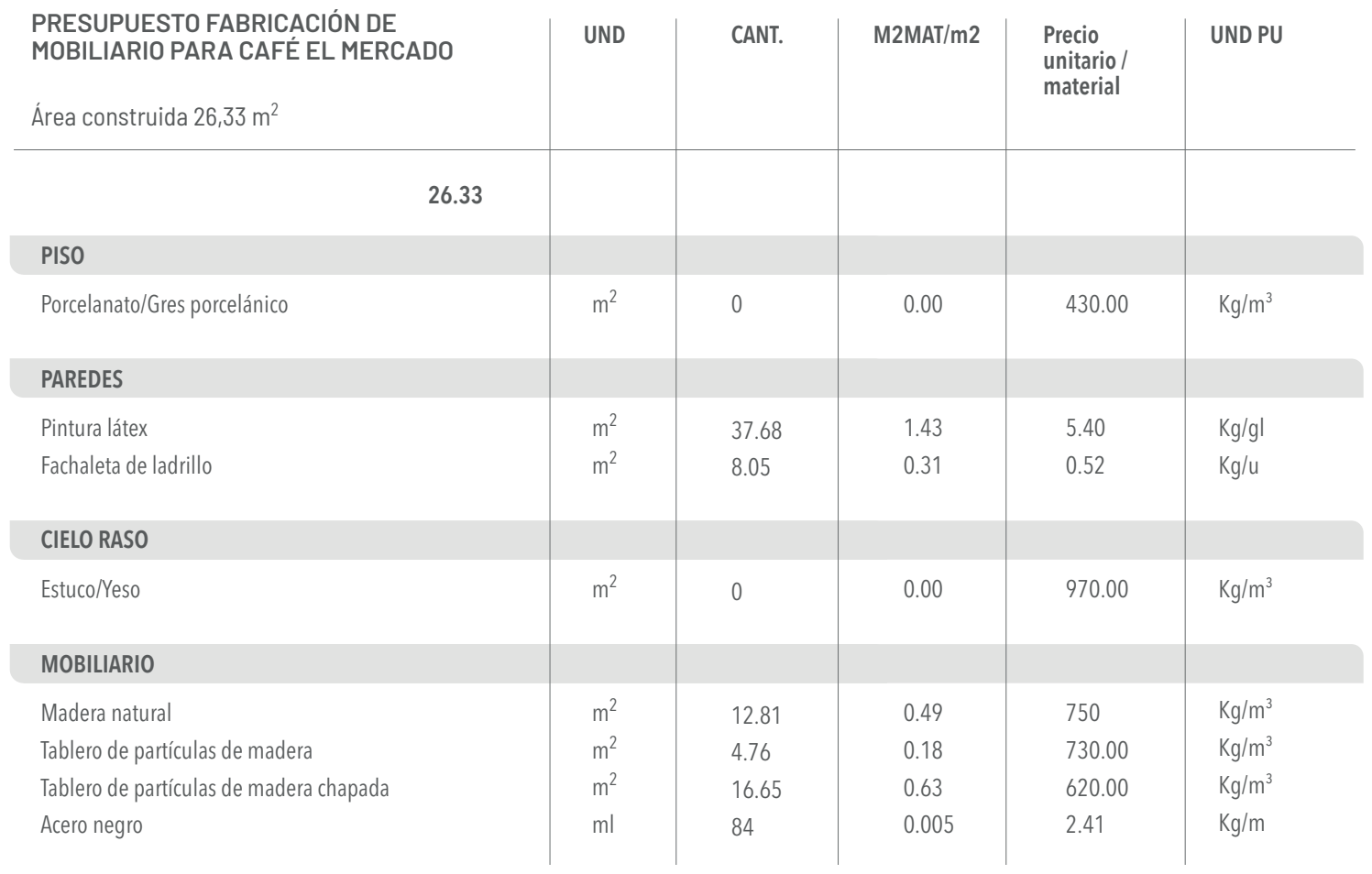

Una vez obtenida la información del peso de los materiales transformamos cada uno a kg, multiplicando la cantidad utilizada por el peso unitario del mismo, tenemos la siguiente tabla. 
Tabla 8. Conversión a kg

\begin{tabular}{|c|c|c|c|c|c|c|c|c|}
\hline $\begin{array}{l}\text { PRESUPUESTO FABRICACIÓN DE } \\
\text { MOBILIARIO PARA CAFÉ EL MERCADO } \\
\text { Área construida } 26,33 \mathrm{~m}^{2}\end{array}$ & UND & CANT. & $\mathrm{M} 2 \mathrm{MAT} / \mathrm{m} 2$ & $\begin{array}{l}\text { Precio } \\
\text { unitario / } \\
\text { material }\end{array}$ & UND PU & Cantidad & $\begin{array}{l}\text { Unidad } \\
\text { origen }\end{array}$ & Peso total \\
\hline 26.33 & & & & & & & & \\
\hline \multicolumn{9}{|l|}{ PISO } \\
\hline Porcelanato/Gres porcelánico & $\mathrm{m}^{2}$ & 0 & 0.00 & 430.00 & $\mathrm{Kg} / \mathrm{m}^{3}$ & & & \\
\hline \multicolumn{9}{|l|}{ PAREDES } \\
\hline $\begin{array}{l}\text { Pintura látex } \\
\text { Fachaleta de ladrillo }\end{array}$ & $\begin{array}{l}\mathrm{m}^{2} \\
\mathrm{~m}^{2}\end{array}$ & $\begin{array}{l}37.68 \\
8.05\end{array}$ & $\begin{array}{l}1.43 \\
0.31\end{array}$ & $\begin{array}{l}5.40 \\
0.52\end{array}$ & $\begin{array}{l}\mathrm{Kg} / \mathrm{gl} \\
\mathrm{Kg} / \mathrm{u}\end{array}$ & $\begin{array}{l}1.50 \\
370.97\end{array}$ & $\begin{array}{l}g l \\
u\end{array}$ & $\begin{array}{l}8.10 \\
192.90\end{array}$ \\
\hline \multicolumn{9}{|l|}{ CIELO RASO } \\
\hline Estuco/Yeso & $\mathrm{m}^{2}$ & 0 & 0.00 & 970.00 & $\mathrm{Kg} / \mathrm{m}^{3}$ & $\mathrm{Kg} / \mathrm{m} 3$ & & \\
\hline \multicolumn{9}{|l|}{ MOBILIARIO } \\
\hline $\begin{array}{l}\text { Madera natural } \\
\text { Tablero de partículas de madera } \\
\text { Tablero de partículas de madera chapada } \\
\text { Acero negro }\end{array}$ & $\begin{array}{l}\mathrm{m}^{2} \\
\mathrm{~m}^{2} \\
\mathrm{~m}^{2} \\
\mathrm{ml}\end{array}$ & $\begin{array}{l}12.81 \\
4.76 \\
16.65 \\
0.13\end{array}$ & $\begin{array}{l}0.49 \\
0.18 \\
0.63 \\
0.005\end{array}$ & $\begin{array}{l}750 \\
730.00 \\
620.00 \\
2.41\end{array}$ & $\begin{array}{l}\mathrm{Kg} / \mathrm{m}^{3} \\
\mathrm{Kg} / \mathrm{m}^{3} \\
\mathrm{Kg} / \mathrm{m}^{3} \\
\mathrm{Kg} / \mathrm{m}\end{array}$ & $\begin{array}{l}0.32 \\
0.09 \\
0.30 \\
84.00\end{array}$ & $\begin{array}{l}\mathrm{m}^{3} \\
\mathrm{~m}^{3} \\
\mathrm{~m}^{3} \\
\mathrm{~m}\end{array}$ & $\begin{array}{l}240.00 \\
62.55 \\
185.85 \\
202.44\end{array}$ \\
\hline
\end{tabular}

Para convertir los materiales consumidos en los proyectos a $\mathrm{kg} / \mathrm{m}^{2}$ construido multiplicamos el peso total de los mismos por el valor obtenido de la división de los $\mathrm{m}^{2}$, correspondientes a los materiales empleados en la ejecución sobre la superficie construida en cada uno de los proyectos, a fin de crear una Base de Datos medioambientales específica en peso $/ \mathrm{m}^{2}$ construido. 
Tabla 9. Conversión a kg/m²

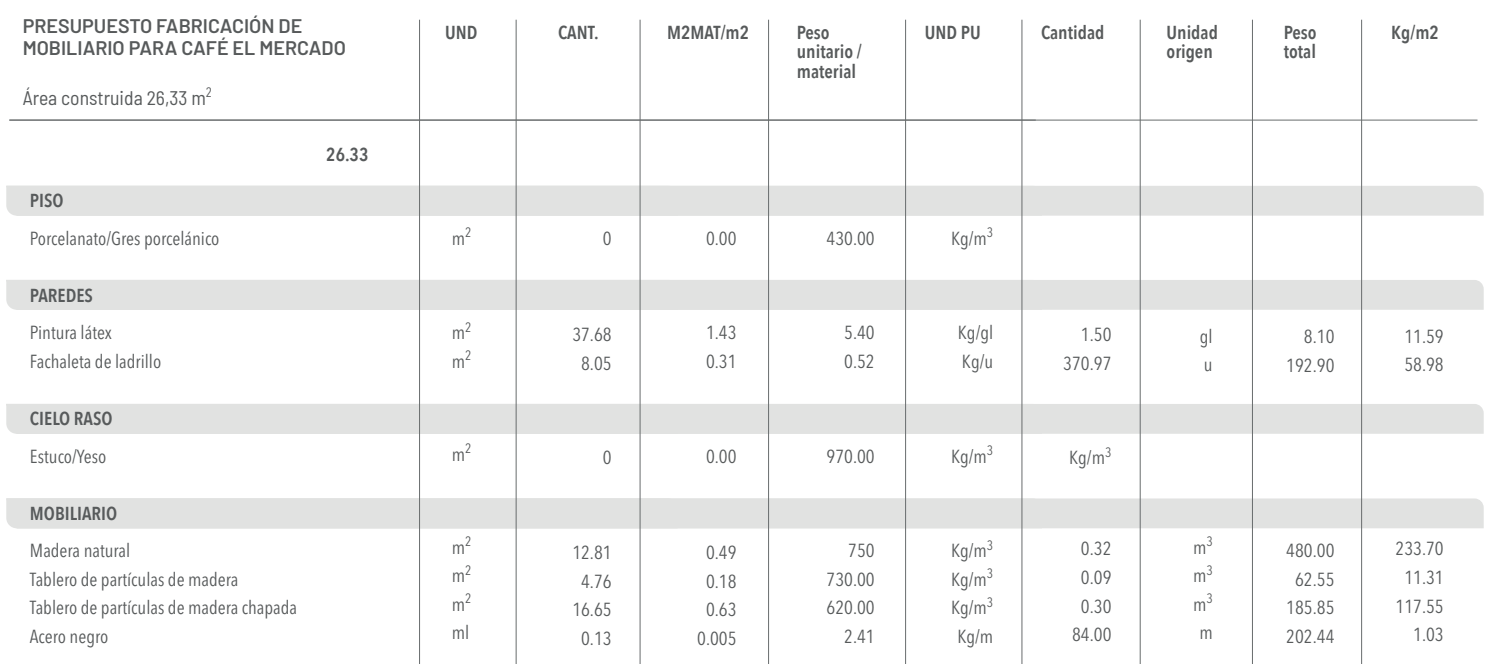

E4.5. Determinación de componentes representativos de la muestra.

Permite sintetizar la información de mayor relevancia; dando lugar a la elección, de forma justificada, de materiales representativos de la muestra, ya que un material con poco peso puede tener un impacto medioambiental relevante. 
Tabla 10. Determinación de componentes representativos de la muestra

\begin{tabular}{|c|c|c|c|c|c|c|c|c|c|}
\hline $\begin{array}{l}\text { PRESUPUESTO FABRICACIÓN DE } \\
\text { MOBILIARIO PARA CAFÉ EL MERCADO } \\
\text { Área construida } 26,33 \mathrm{~m}^{2}\end{array}$ & UND & CANT. & $\mathrm{M} 2 \mathrm{MAT} / \mathrm{m} 2$ & $\begin{array}{l}\text { Peso } \\
\text { unitario/ } \\
\text { material }\end{array}$ & UND PU & Cantidad & $\begin{array}{l}\text { Unidad } \\
\text { origen }\end{array}$ & $\begin{array}{l}\text { Peso } \\
\text { total }\end{array}$ & $\mathrm{Kg} / \mathrm{m} 2$ \\
\hline 26.3 & & & & & & & & & \\
\hline \multicolumn{10}{|l|}{ PISO } \\
\hline Porcelanato/Gres porcelánico & $\mathrm{m}^{2}$ & 0 & 0.00 & 430.00 & $\mathrm{Kg} / \mathrm{m} 3$ & & & & \\
\hline \multicolumn{10}{|l|}{ PAREDES } \\
\hline Pintura látex & $\mathrm{m}^{2}$ & 37.68 & 1.43 & 5.40 & $\mathrm{Kg} / \mathrm{gl}$ & 1.50 & gl & 8.10 & 11.59 \\
\hline Fachaleta de ladrillo & $\mathrm{m}^{2}$ & 8.05 & 0.31 & 0.52 & $\mathrm{Kg} / \mathrm{u}$ & 370.97 & u & 192.90 & 58.98 \\
\hline Mortero prefabricado & $\mathrm{m}^{2}$ & 8.05 & 0.31 & 1.900 .00 & $\mathrm{Kg} / \mathrm{m}^{3}$ & 0.08 & $\mathrm{~m}^{3}$ & 152.95 & 46.76 \\
\hline \multicolumn{10}{|l|}{ CIELO RASO } \\
\hline Estuco/Yeso & $\mathrm{m}^{2}$ & 0 & 0.00 & 970.00 & $\mathrm{Kg} / \mathrm{m}^{3}$ & & & & \\
\hline \multicolumn{10}{|l|}{ MOBILIARIO } \\
\hline Madera natural & $\mathrm{m}^{2}$ & 12.81 & 0.49 & 750 & $\mathrm{Kg} / \mathrm{m}^{3}$ & 0.32 & $\mathrm{~m}^{3}$ & 240.00 & 116.76 \\
\hline Tablero de partículas de madera & $\mathrm{m}^{2}$ & $\begin{array}{r}12.01 \\
4.76\end{array}$ & 0.18 & 730 & $\mathrm{Kg} / \mathrm{m}^{3}$ & 0.09 & $\mathrm{~m}^{3}$ & 62.55 & 11.31 \\
\hline Tablero de partículas de madera chapada & $\mathrm{m}^{2}$ & 16.65 & 0.63 & 620 & $\mathrm{Kg} / \mathrm{m}^{3}$ & 0.30 & $\mathrm{~m}^{3}$ & 185.85 & 117.55 \\
\hline Cola natural & $\mathrm{m}^{2}$ & 34.22 & 1.30 & 1.10 & $\mathrm{Kg} / \mathrm{l}$ & 1.50 & I & 1.65 & 2.14 \\
\hline Fondo Blanco/Pintura acrílica & $\mathrm{m}^{2}$ & 4.76 & 0.18 & 1.80 & $\mathrm{Kg} / \mathrm{l}$ & 1.25 & 1 & 2.25 & 0.41 \\
\hline Barniz & $\mathrm{m}^{2}$ & $\begin{array}{r}4.10 \\
17.57\end{array}$ & 0.67 & 3.95 & $\mathrm{Kg} / \mathrm{gl}$ & 3.03 & gl & 11.97 & 7.99 \\
\hline Acero negro & $\mathrm{m}^{2}$ & 0.13 & 0.005 & 2.41 & $\mathrm{Kg} / \mathrm{m}$ & 84.00 & $\mathrm{~m}$ & 202.44 & 1.03 \\
\hline
\end{tabular}

E4.6. Determinación de consumo energético y emisiones de $\mathrm{CO}_{2}$ de los materiales.

En esta etapa se determinó los parámetros medioambientales correspondientes a emisiones de $\mathrm{CO}_{2}$ y consumo energético que se producen en la fabricación de cada material definido, cuyos datos se expresan en $\mathrm{MJ} / \mathrm{kg}$ y $\mathrm{kgCO}_{2} / \mathrm{kg}$ respectivamente. Para esto se multiplicó el valor del peso total en $\mathrm{kg}$ por el valor correspondiente de consumo energético y emisiones de $\mathrm{CO}_{2}$ de cada material.

Hay que tener en cuenta que la fiabilidad de los datos está muy relacionada con las posibilidades de acceder a fuentes de información precisas, que no siempre están disponibles; y debido a que en nuestro país estos datos son limitados o nulos se pueden usar bases de datos como referencias aproximadas.
Se tomó como referencia principal la base de datos española (BEDEC) del Instituto de Tecnología de la Construcción de Cataluña (ITeC) y la base de datos Inventory of Carbon \& Energy (ICE), desarrollada por la University of Bath del Reino Unido.

De igual manera, dado que algunos materiales que aparecen en los proyectos carecen de esta información medioambiental se utilizarán, como referencias, materiales con similares características, como es el caso de la laca y fondo para madera. 
Tabla 11. Determinación de consumo energético y emisiones de $\mathrm{CO}_{2}$ de los materiales

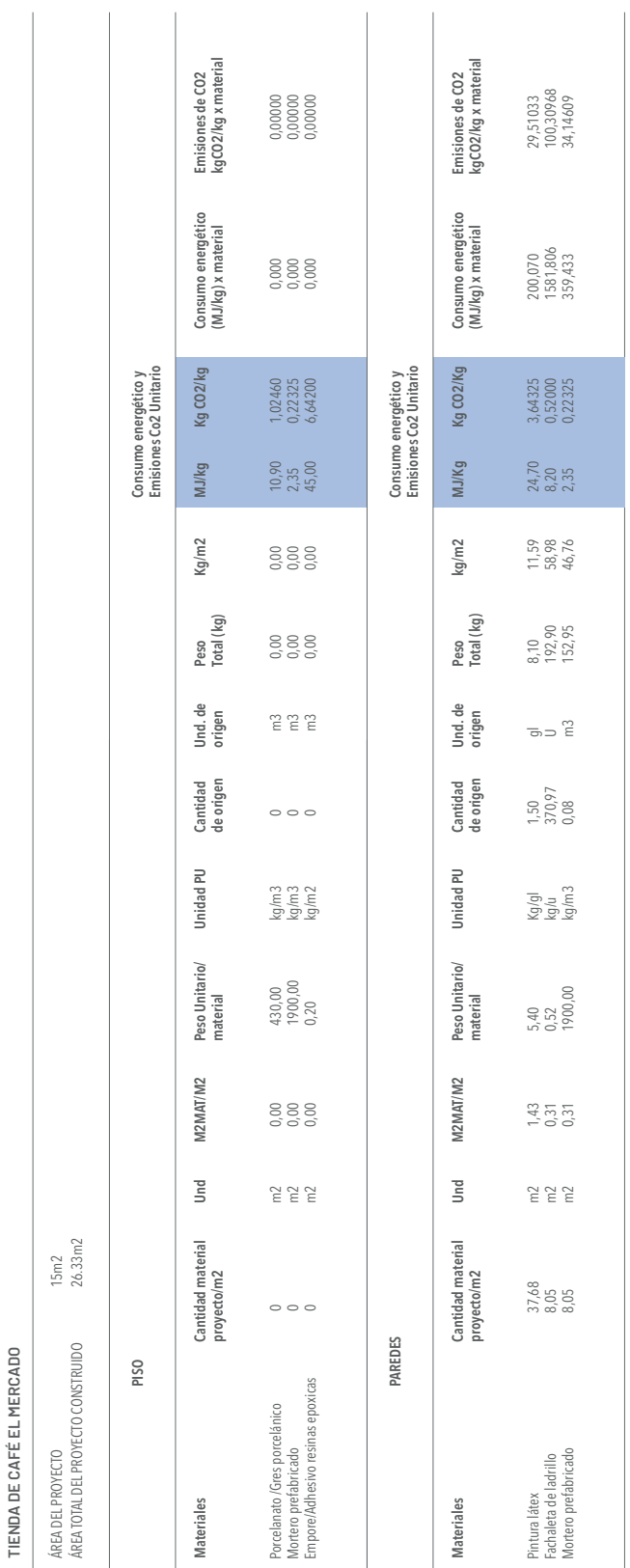

E4.7. Consumo por $\mathrm{m}^{2}$ construido

Para determinar el consumo energético y las emisiones de $\mathrm{CO}_{2}$ por $\mathrm{m}^{2}$ construido, correspondiente a cada uno de los materiales de los proyectos,

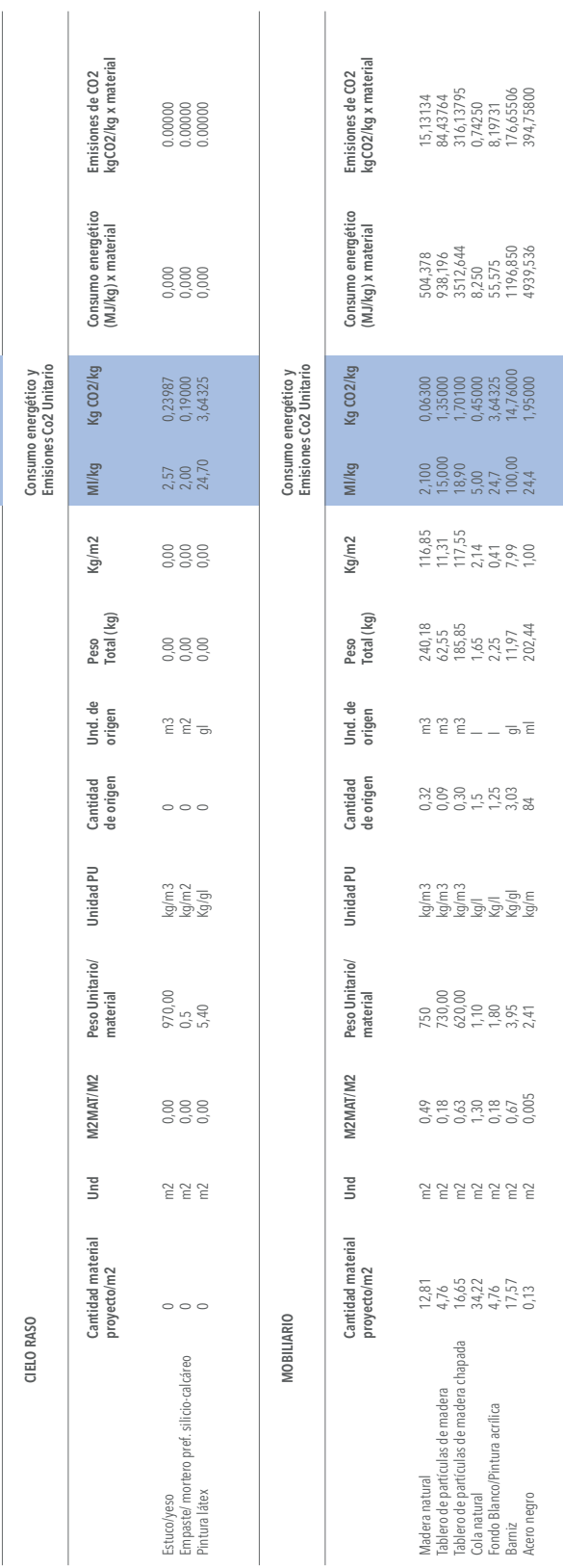

se multiplica los resultados obtenidos en $\mathrm{kg} / \mathrm{m}^{2}$ por los valores medioambientales de cada material, obteniendo así los resultados deseados expresados en $\mathrm{MJ} / \mathrm{m}^{2}$ y kgCO . 
Tabla 12. Consumo por $\mathrm{m}^{2}$ construido

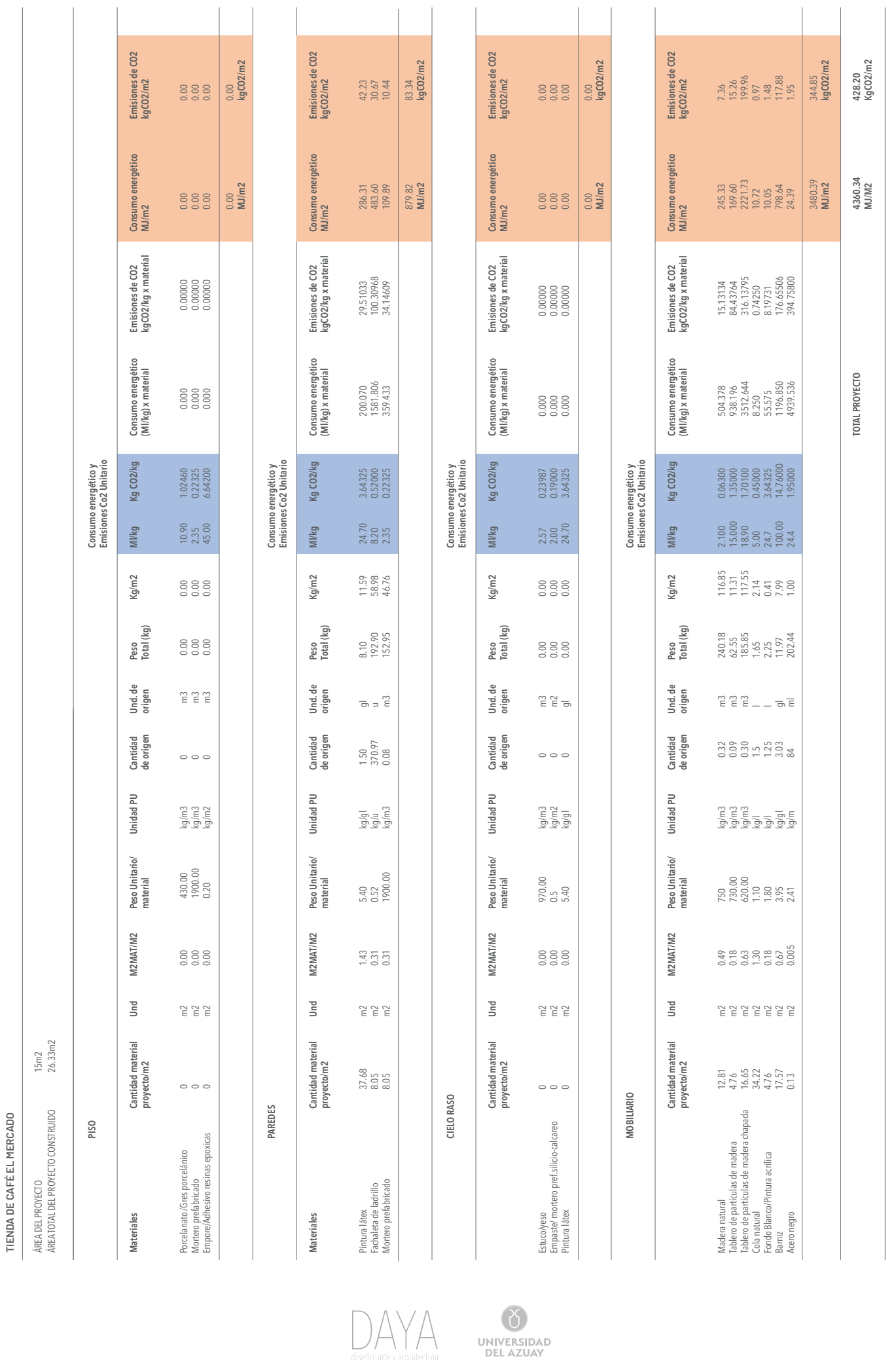


Los proyectos seleccionados para esta etapa son:

- Tienda de Café el Mercado

- Qala Restaurante

- Serafina Bistro

\section{Tienda de Café el Mercado}

Ubicada en el centro histórico de la ciudad de Cuenca, Calle Larga 8-27 y Luis Cordero.

Figura 5. Fotografía Tienda Café el Mercado

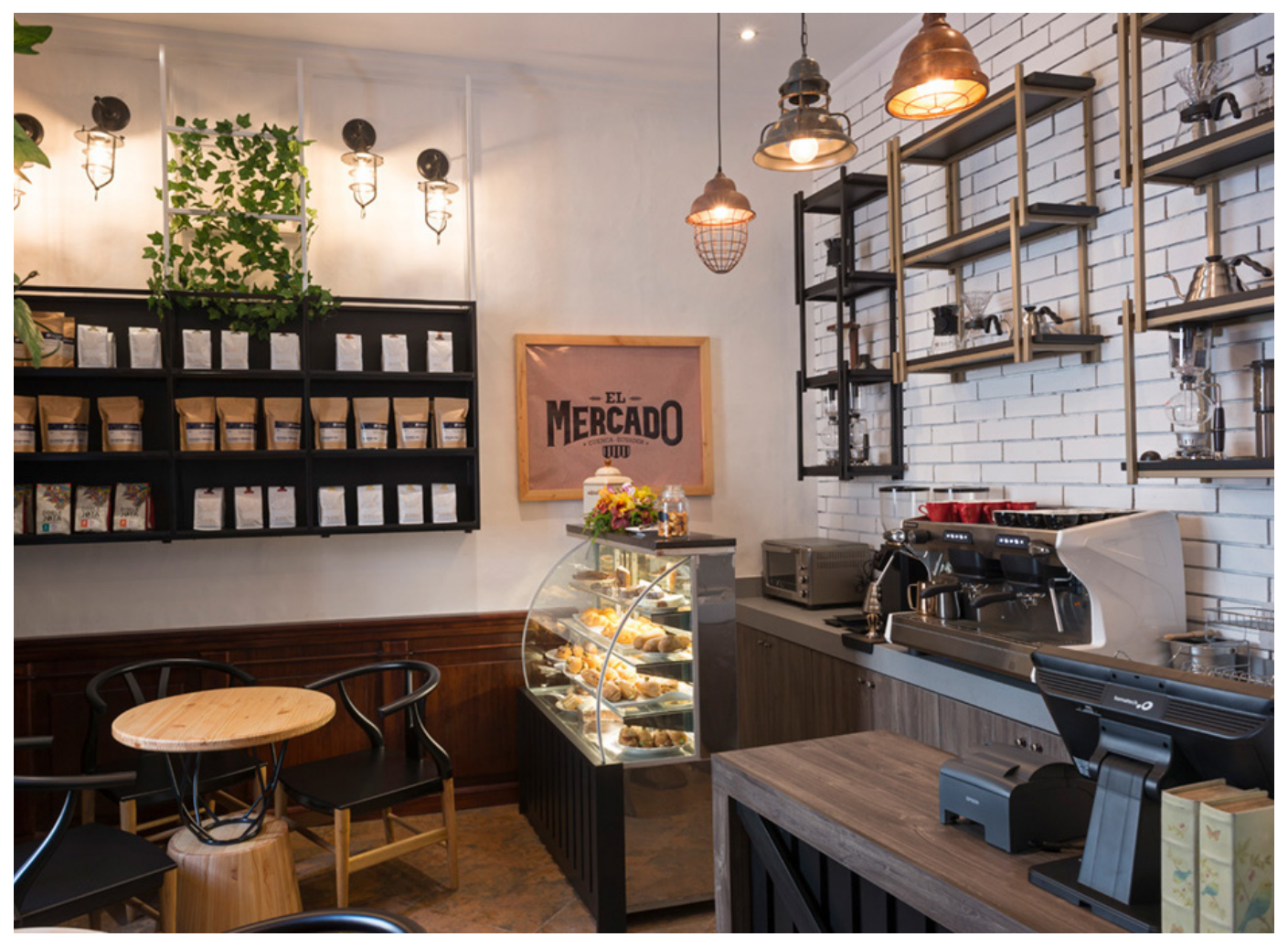

De Carrión, (2018). 


\section{Qala Restaurante}

Ubicado en la Paseo 3 de Noviembre, Cuenca-Ecuador.

Figura 6. Fotografía Qala Restaurante

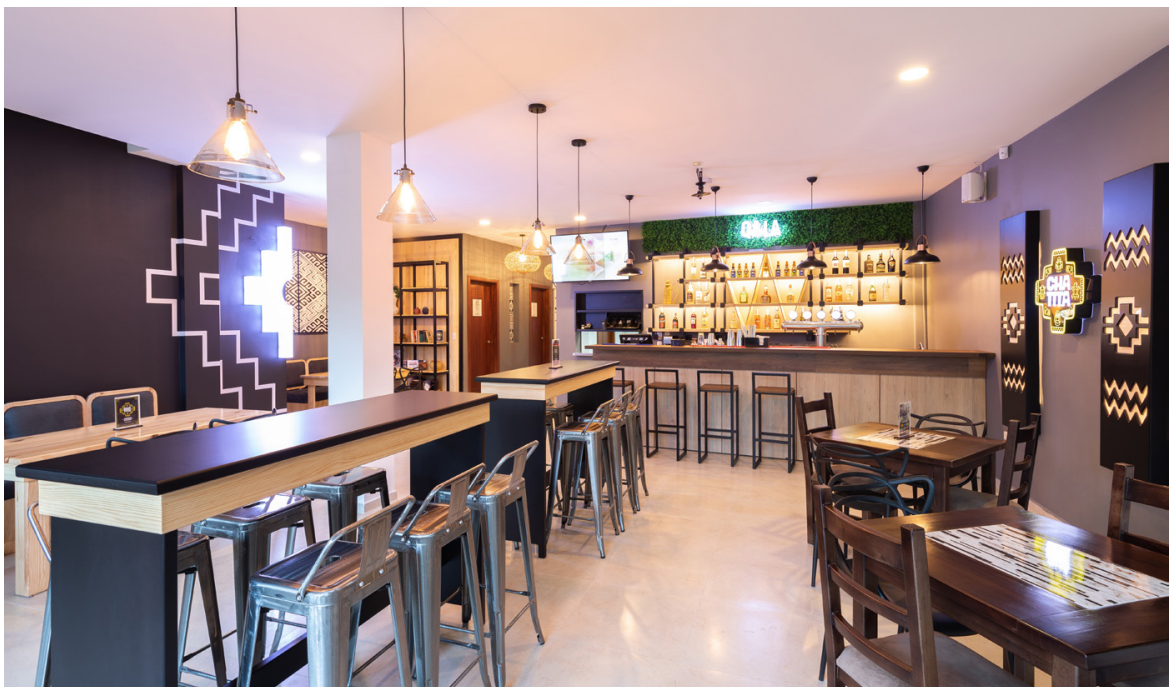

De Provoste, (2019).

\section{Serafina Bistro}

Ubicado en la Roberto Crespo Toral y Miguel Cordero Dávila.

Figura 7. Fotografía Serafina Bistro

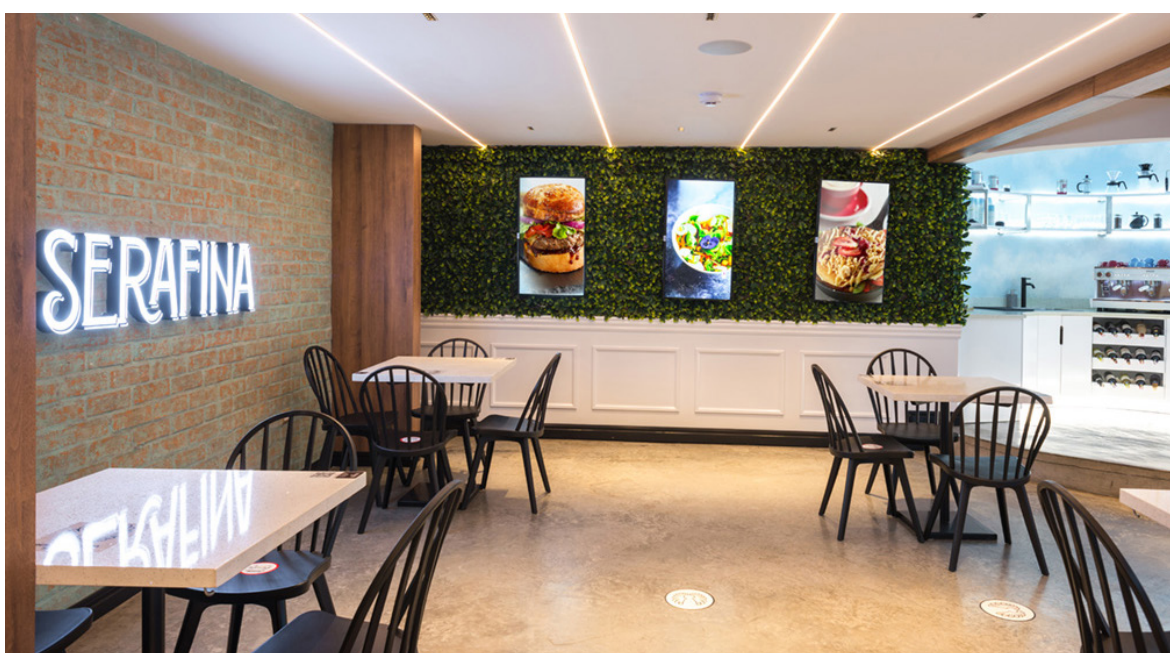

De Carrión, (2020). 


\section{Resultados}

En la Tienda de Café el mercado considerando que los materiales de la envolvente, piso, paredes y cielo raso se conservaron, se intervino con revestimientos de paredes y mobiliario, obteniendo un impacto de $4360.34 \mathrm{MJ} / \mathrm{m}^{2}$ y $428.20 \mathrm{kgCO} / \mathrm{m}^{2}$ de consumo energético y emisiones de los materiales en su proceso de fabricación.

En Qala Restaurante, de igual manera, siguiendo el mismo proceso y conservando los materiales de la envolvente, piso, cielo raso y paredes, así como parte del mobiliario existente, se obtuvo un impacto en cuanto al consumo energético de los materiales en su proceso de fabricación de 2132.14 MJ/ $\mathrm{m}^{2}$ y $258.89 \mathrm{kgCO} / \mathrm{m}^{2}$ de emisiones en su proceso de fabricación.

En Serafina Bistro, al tratarse de una intervención en un espacio completamente remodelado, en donde se utilizaron materiales nuevos en su mayoría, el impacto en cuanto al consumo energético de los materiales en su proceso de fabricación fue de 24.718.36 MJ/m $2349.59 \mathrm{kgCO} / \mathrm{m}^{2}$ de emisiones en su proceso de fabricación.

Para reducir el impacto medio ambiental, sin comprometer el diseño interior, es indispensable tener en cuenta varias alternativas de materiales para tomar decisiones acertadas al momento de seleccionarlas y utilizarlas, generando así propuestas sustentables.

Los materiales naturales son una buena opción si son extraídos de manera responsable, así como contemplar materiales que se obtienen de materias primas de origen local.
Otro factor importante para disminuir, significativamente, el impacto medio ambiental es conservar materiales que no requieran de grandes intervenciones para recuperarlos, así como la reutilización de lo existente y mantener el diseño lo más simple posible.

Cabe aclarar que se tuvo cierta flexibilidad, por tratarse de un estudio experimental, el utilizar materiales habituales en intervenciones de diseño interior comercial en restaurantes y cafeterías en la ciudad de Cuenca; pero la posibilidad de utilizar en otros proyectos, materiales preferiblemente ambientales o materiales ecológicos, podría mejorar aún más los resultados obtenidos, reduciendo el impacto sobre la salud de las personas y sobre el medio ambiente.

Se ha podido determinar también que, la conservación de materiales que no requieren de mayor intervención para recuperarlos aporta, de manera positiva, en cada proyecto. 
Tabla 13. Resultados Tienda de café El Mercado

\begin{tabular}{|lll|}
\hline TOTAL CONSERVANDO MATERIALES & $\begin{array}{l}4360.34 \\
\mathrm{MJ} / \mathrm{m}^{2}\end{array}$ & $\begin{array}{l}428.20 \\
\mathrm{kgCO} / \mathrm{m}^{2}\end{array}$ \\
\hline & & \\
\hline $\begin{array}{l}\text { TOTAL REEMPLAZANDO MATERIALES } \\
\text { CON MENOR IMPACTO }\end{array}$ & $\begin{array}{ll}1653.22 \\
\mathrm{MJ} / \mathrm{m}^{2}\end{array}$ & $\begin{array}{l}144.11 \\
\mathrm{kgCO} / \mathrm{m}^{2}\end{array}$ \\
\hline & & \\
\hline & & \\
\hline
\end{tabular}

Figura 8. Total, consumo energético y emisiones de $\mathrm{CO}_{2}$ de materiales utilizados
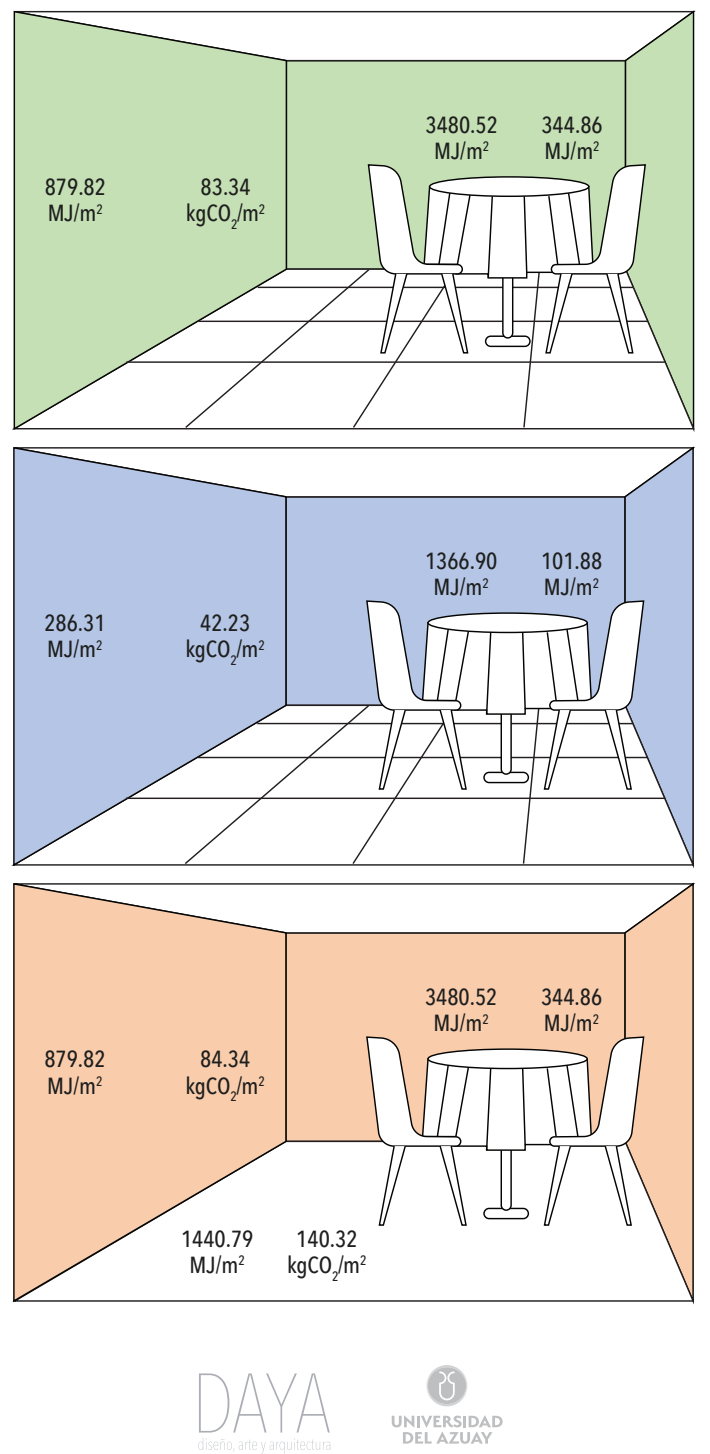
Figura 9. Renders proyecto, Tienda de Café El Mercado

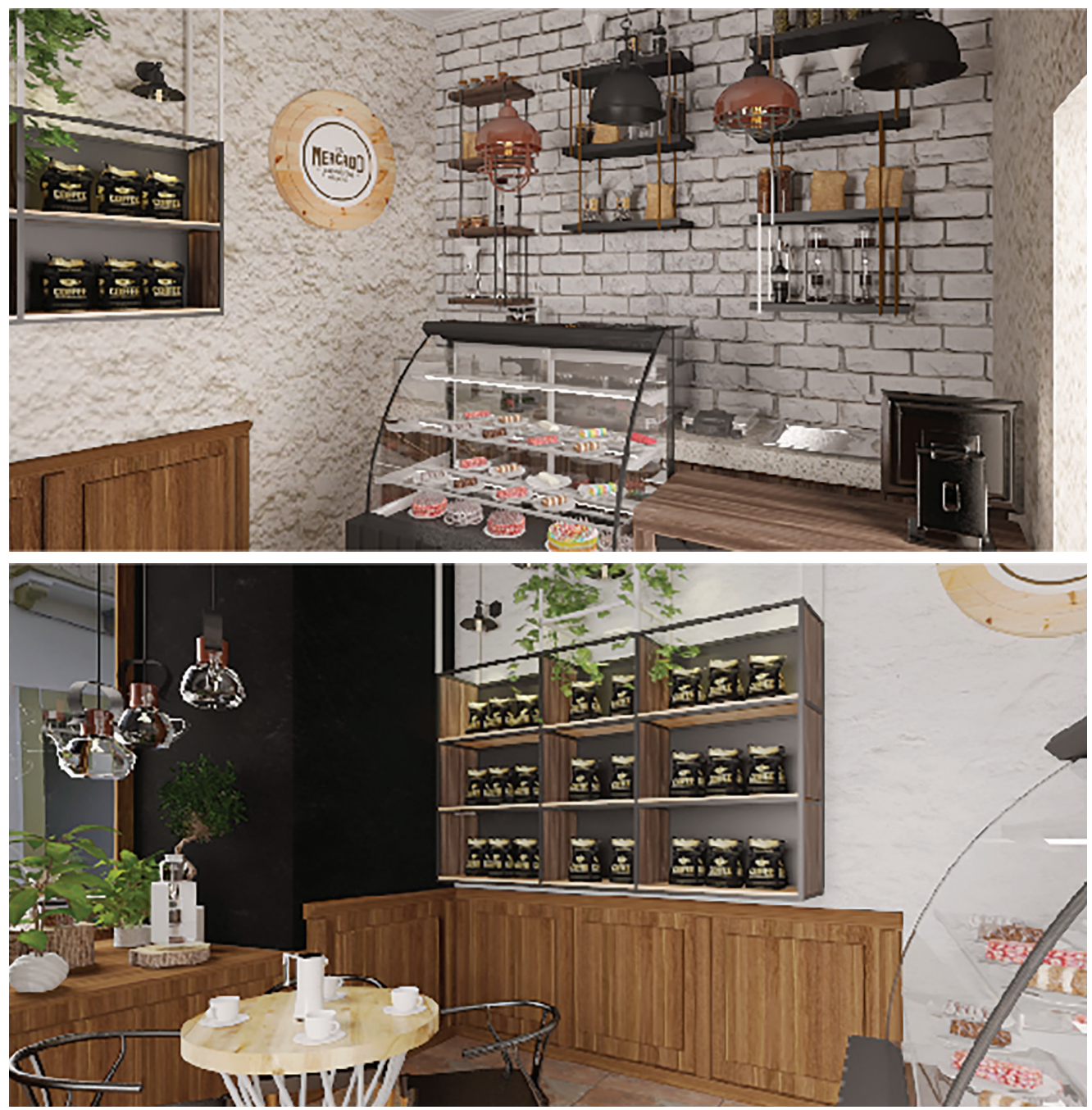

De Vélez, (2020). 
Figura 10. Renders propuesta cambio materiales, Tienda de Café El Mercado
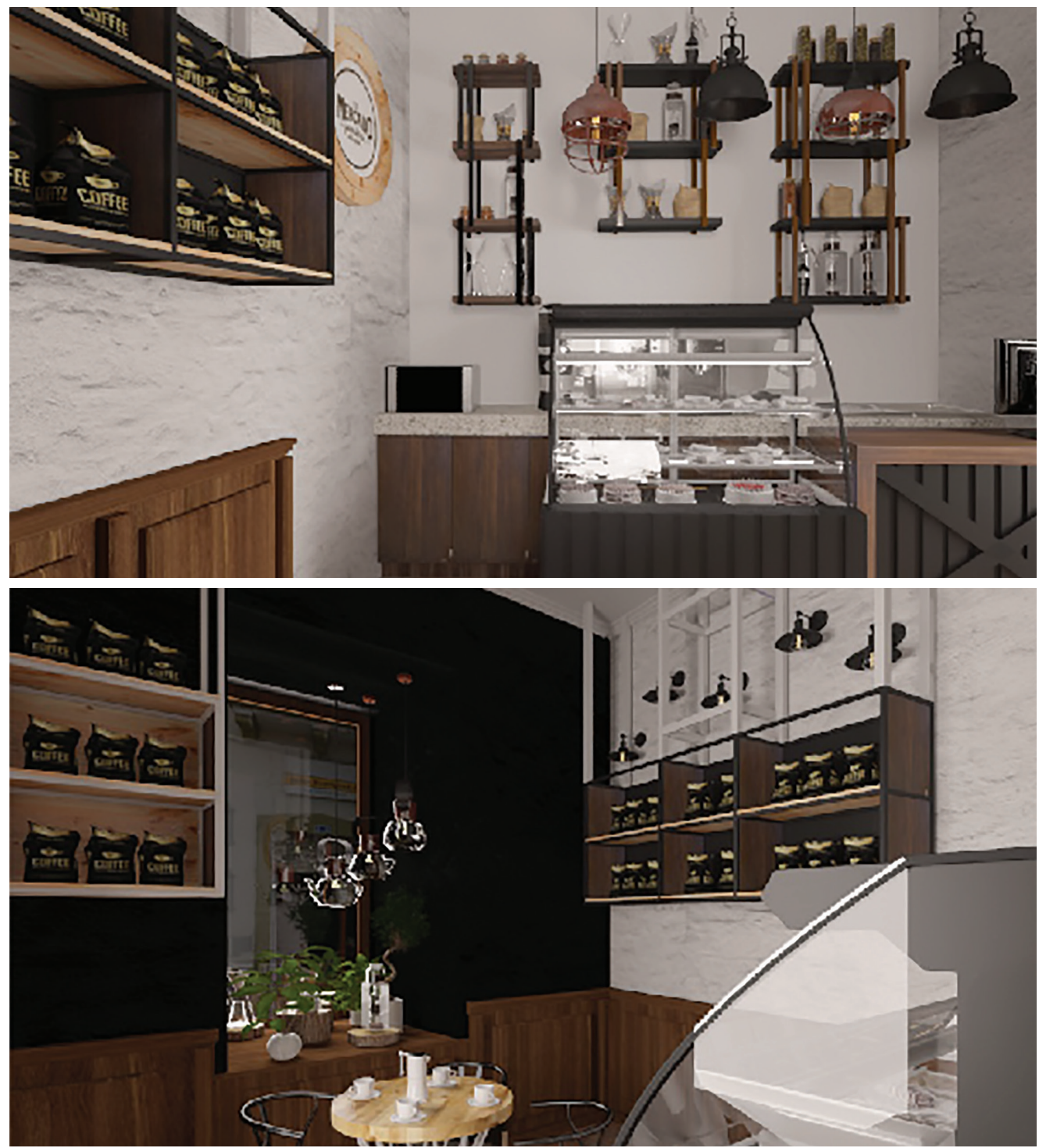

De Vélez, (2020).

Como se puede evidenciar en los resultados obtenidos, al conservar materiales se obtiene un impacto de $4360.34 \mathrm{MJ} / \mathrm{m}^{2}$ y $428.20 \mathrm{kgCO} / \mathrm{m}^{2}$.

Si se reemplazan algunos materiales por otros de menor impacto se logra reducir 1653.22 $\mathrm{MJ} / \mathrm{m}^{2}$ y $144.11 \mathrm{kgCO} / \mathrm{m}^{2}$.
Por eso es importante conservar materiales y utilizar aquellos con menor impacto, ya que al intervenir los espacios en su totalidad hay un incremento de $6273.73 \mathrm{MJ} / \mathrm{m}^{2}$ y $614.70 \mathrm{kgCO} / \mathrm{m}^{2}$. 
Tabla 14. Resultados Qala Restaurante

\begin{tabular}{|lll|}
\hline TOTAL CONSERVANDO MATERIALES & $\begin{array}{ll}2132.02 \\
\mathrm{MJ} / \mathrm{m}^{2}\end{array}$ & $\begin{array}{l}258.88 \\
\mathrm{kgCO} / \mathrm{m}^{2}\end{array}$ \\
& & \\
\hline & & \\
\hline $\begin{array}{l}\text { TOTAL REEMPLAZANDO MATERIALES } \\
\text { CON MENOR IMPACTO }\end{array}$ & $\begin{array}{l}774.76 \\
\mathrm{MJ} / \mathrm{m}^{2}\end{array}$ & $\begin{array}{l}84.78 \\
\mathrm{kgCO} / \mathrm{m}^{2}\end{array}$ \\
& & \\
\hline & & \\
\hline & 38078 & 3399.15 \\
$\mathrm{MJ} / \mathrm{m}^{2}$ & $\mathrm{kgCO} / \mathrm{m}^{2}$ \\
\hline
\end{tabular}

Figura 11. Total, consumo energético y emisiones de $\mathrm{CO}_{2}$ de materiales utilizados
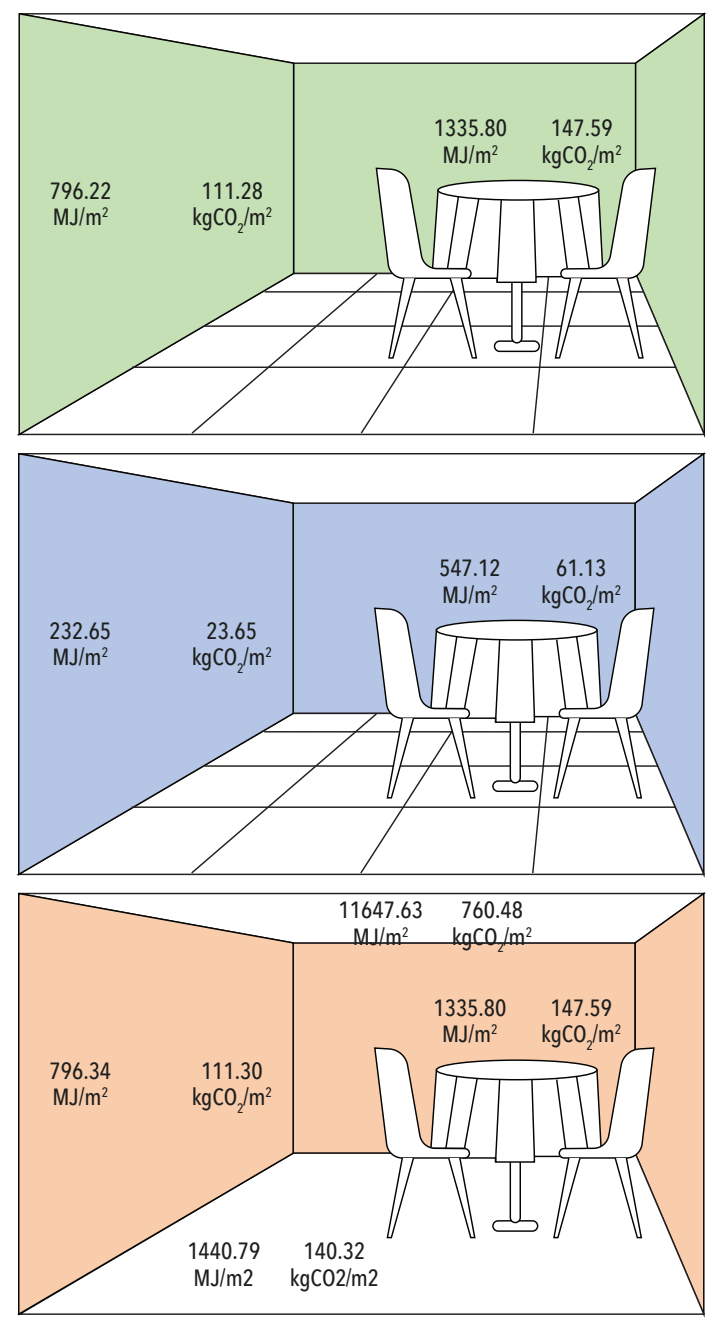
Figura 12. Renders proyecto, Oala Restaurante

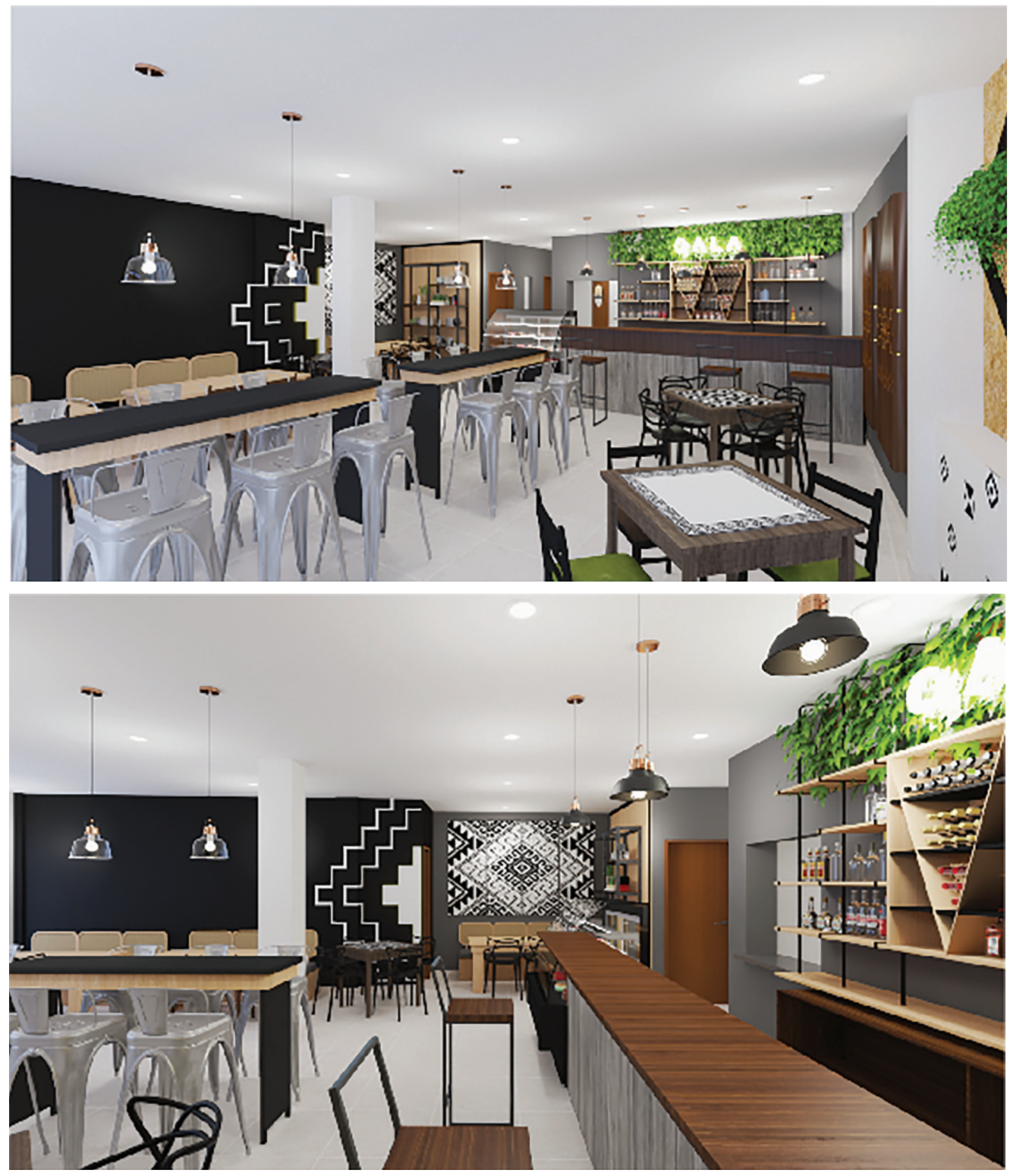


Figura 13. Renders proyecto, Qala Restaurante
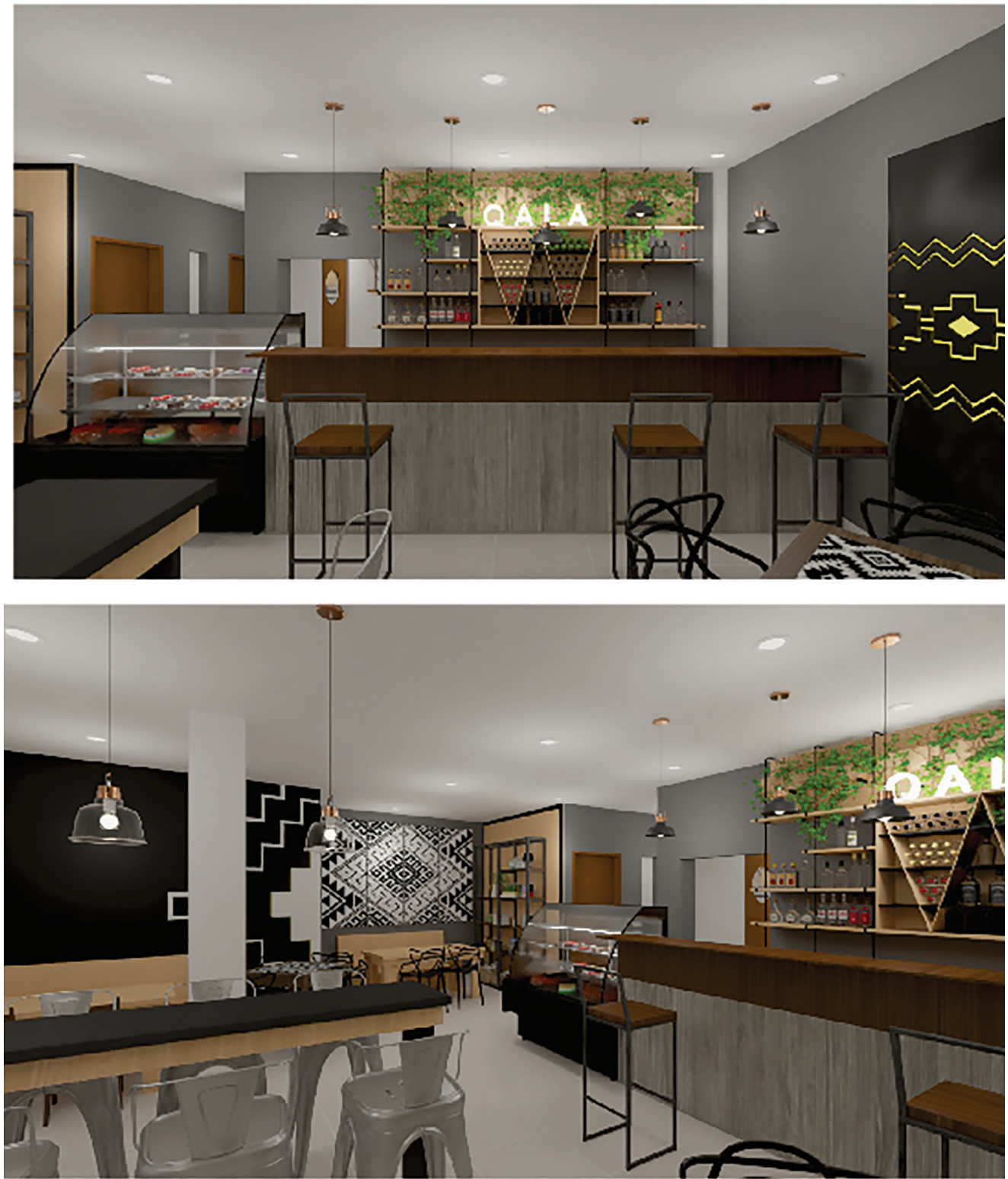

De igual manera, con la conservación de materiales los impactos generados en este proyecto son $2132.02 \mathrm{MJ} / \mathrm{m}^{2}$ y $258.88 \mathrm{kgCO} / \mathrm{m}^{2}$.
Si lo reemplazamos por materiales de menor impacto los resultados disminuyen a 774.76 $\mathrm{MJ} / \mathrm{m}^{2}$ y $84.78 \mathrm{kgCO} / \mathrm{m}^{2}$. Y si la intervención fuera en su totalidad el incremento del impacto sería de $38.078 .53 \mathrm{MJ} / \mathrm{m}^{2}$ y $3399.15 \mathrm{kgCO} / \mathrm{m}^{2}$. 
Tabla 15. Resultados Qala Restaurante

\begin{tabular}{|c|c|c|}
\hline $\begin{array}{l}\text { TOTAL REEMPLAZANDO MATERIALES } \\
\text { CON MENOR IMPACTO }\end{array}$ & $\begin{array}{l}16068.01 \\
\mathrm{MJ} / \mathrm{m}^{2}\end{array}$ & $\begin{array}{l}1287.37 \\
\mathrm{kgCO} / \mathrm{m}^{2}\end{array}$ \\
\hline TOTAL MATERIALES NUEVOS & $\begin{array}{l}24718.36 \\
\mathrm{MJ} / \mathrm{m}^{2}\end{array}$ & $\begin{array}{l}2349.59 \\
\mathrm{kgCO} / \mathrm{m}^{2}\end{array}$ \\
\hline
\end{tabular}

Figura 14. Total, consumo energético y emisiones de $\mathrm{CO}_{2}$ de materiales utilizados

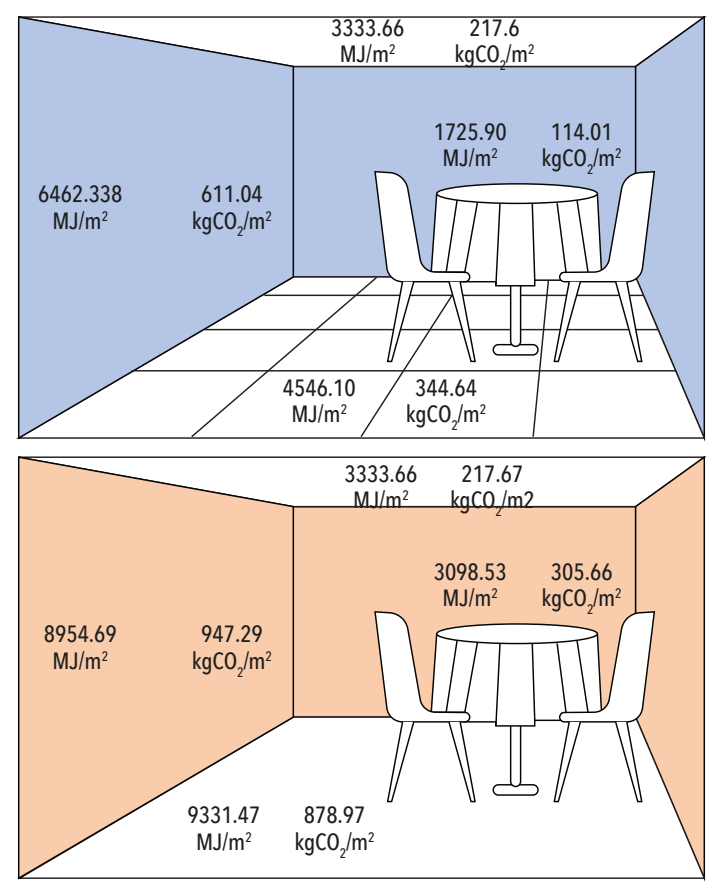


Figura 15. Renders proyecto, Serafina Bistro
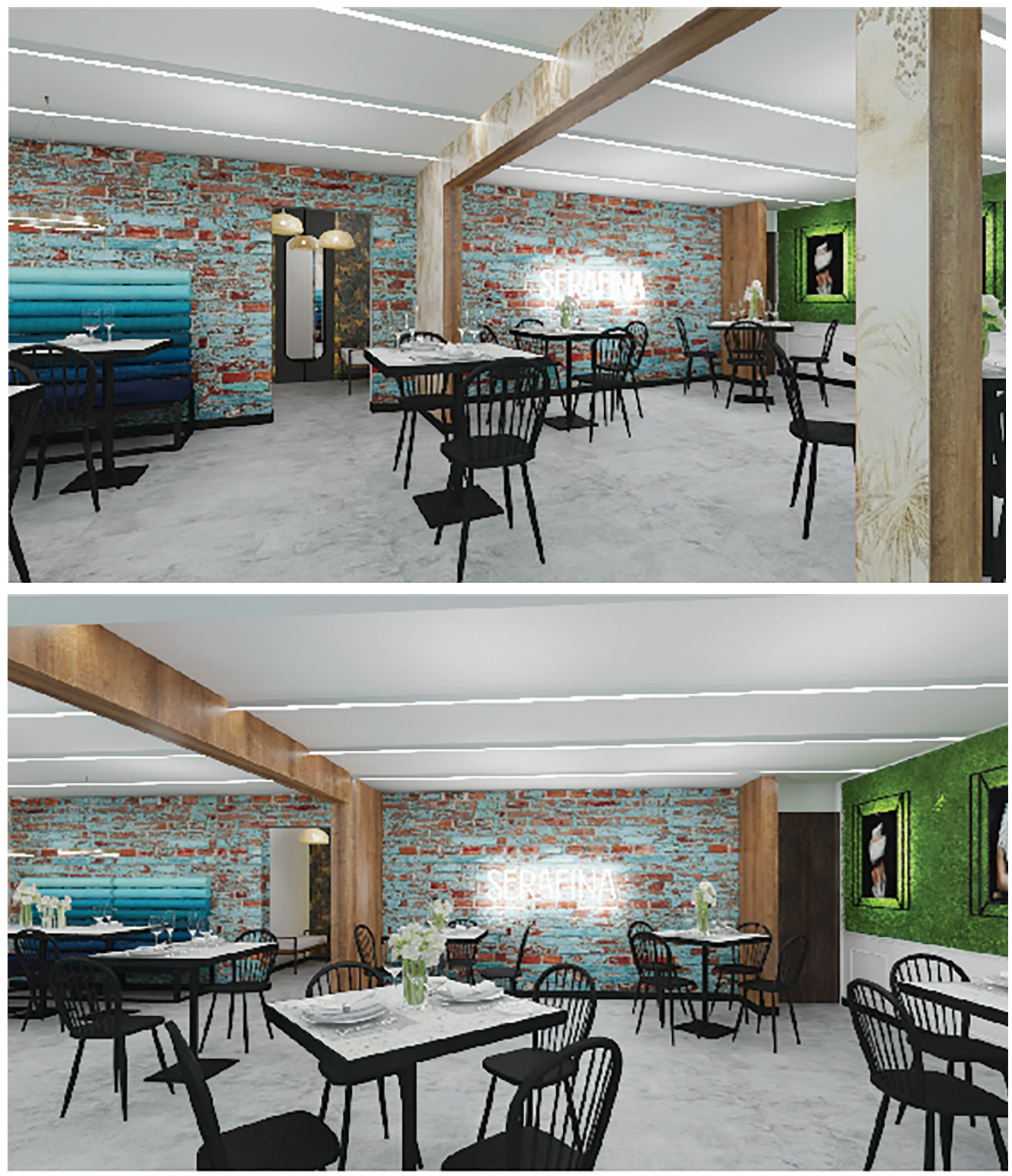
Figura 16. Renders propuesta cambio materiales, Serafina Bistro

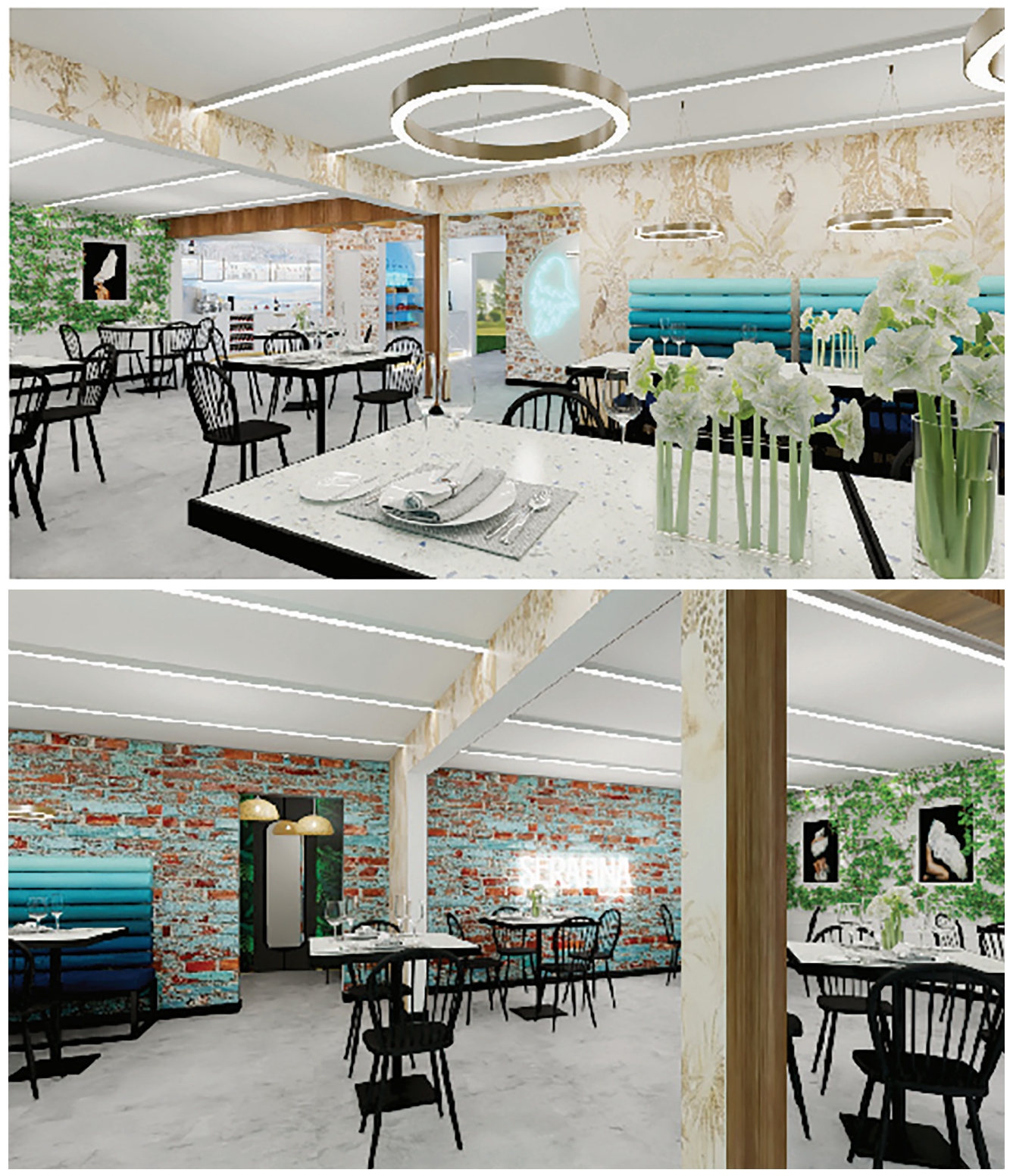


En este proyecto, al tratarse de una intervención casi en su totalidad con materiales nuevos el impacto es alto $24718.36 \mathrm{MJ} / \mathrm{m}^{2}$ y $2349.59 \mathrm{~kg} \mathrm{CO} / \mathrm{m}^{2}$.

$\mathrm{Si}$ se reemplazan algunos materiales por otros con menor impacto se puede reducir $16068.01 \mathrm{MJ} / \mathrm{m}^{2}$ y $1287.37 \mathrm{~kg} \mathrm{CO} / \mathrm{m}^{2}$
Es nuestra responsabilidad, como profesionales en el ámbito del diseño, contribuir de manera innovadora a reducir los impactos medioambientales y generar espacios que promuevan el bienestar de los ocupantes y del medioambiente.

Con la selección adecuada de materiales y el diseño consciente se pueden reducir los impactos medioambientales.

\section{Conclusiones}

Los resultados de esta investigación muestran las posibilidades y beneficios de la adecuada selección de materiales para las intervenciones de espacios interiores comerciales, logrando disminuir la cantidad de energía consumida y emisiones de $\mathrm{CO}_{2}$, procedentes del proceso de fabricación.

De igual manera reconocer, desde la óptica de la fabricación de materiales, junto a la responsabilidad de generar proyectos con impacto en el medio ambiente y del cual somos responsables, la importancia que tiene nuestra profesión como diseñadores y gestores de recursos en el proceso constructivo, lo que ha permitido desarrollar la presente investigación para aportar hacia la vía de la sostenibilidad.

El modelo generado en la investigación ha dado cumplimiento al objetivo principal: cuantificar el consumo energético y emisiones de $\mathrm{CO}_{2}$ de los materiales utilizados en las intervenciones interiores comerciales, de restaurantes y cafeterías. Siendo éste una herramienta metodológica, de fácil manejo, como alternativa al uso de programas informáticos especializados, que no siempre están disponibles.

Asimismo, se logró desarrollar una base de datos de materiales habituales, utilizados en las intervenciones interiores de restaurantes y cafeterías en la ciudad de Cuenca, con indicadores de consumo energético y emisiones de $\mathrm{CO}_{2}$ referenciales, expresados en $\mathrm{MJ} / \mathrm{kg}$ y $\mathrm{kgCO} / 2 \mathrm{~kg}$, respectivamente, destinada a facilitar la selección de materiales en pro de disminuir los impactos medio ambientales en las intervenciones de diseño interior.

Los límites que la investigación ha encontrado en su desarrollo son:

En algunos casos, pese a la utilización de materiales habituales en intervenciones de espacios comerciales sin comprometer el diseño original de los espacios, no se alcanzó a disminuir significativamente los impactos medioambientales.

Tampoco se ha podido extender el análisis de la energía consumida y emisiones de $\mathrm{CO}_{2}$, como derivados del transporte de materiales, construcción, mantenimiento y comportamiento de residuos, debido al tiempo y a su complejidad.

El tema de materiales sustentables es importante para el sector del diseño y la construcción que pueden abrirse nuevas líneas de investigación a partir de la presente, como:

1. Proponer una metodología de análisis de energía consumida y emisiones de $\mathrm{CO}_{2}$, derivados del transporte de materiales, mantenimiento e instalación de los mismos y comportamiento como residuo.

2. Generar una base de datos más amplia que incorpore materiales ecológicos disponibles en nuestro medio.

3. Generando un catálogo de proveedores y materiales ecológicos disponibles en nuestro medio, que pudieran ser de ayuda para la utilización dentro de los espacios interiores.

4. Generar una base de datos local y nacional, con indicadores medioambientales. 


\section{Referencias}

Arroyo, F.; Bravo, D.; y Rivera, M. (2018). "Economía circular: un camino hacia un Quito más sostenible". INNOVA Research Journal, 3(11), 139-158. https://doi.org/10.33890/innova.v3.n11.2018.767

Corral, P. (2017). "Selección de Materiales para un Interiorismo Sustentable". Revista Interiorgráfico de la división de arquitectura arte y diseño de la universidad de Guanajuato. https://www. interiorgrafico.com/edicion/decimo-septima-edicion-diciembre-2017/seleccion-de-materiales-para-un-interiorismo-sustentable

Daza, P. (2010). "Construcción sostenible de edificios: una alternativa responsable para el desarrollo urbano de Quito". Pontificia Universidad Católica del Ecuador. Tesis previa a la obtención del título de Ingeniero Civil. http://repositorio.puce.edu.ec/bitstream/handle/22000/3603/T-PUCE 3613.pdf?sequence=1\&isAllowed=y

García, J.; Quito, J. y Perdomo, J. (2019). "Análisis de la huella de carbono en la construcción y su impacto sobre el ambiente". Universidad Cooperativa de Colombia, Villavicencio, Ingeniería Civil. http://hdl.handle.net/20.500.12494/16031

García, W. (2016). "Primer Informe Bienal de Actualización del Ecuador a la Convención Marco de las Naciones Unidas sobre el Cambio Climático". Resumen Ejecutivo. https://info.undp.org/ docs/pdc/Documents/ECU/IBA\%20ECU\%20resumen\%20ejecutivo.pdf

Garrido, L. (s.f.). "Hacia una arquitectura ecológica". http://www.arqgea.com/docs/ARQGEA_Hacia_una_arquitectura_ecol\%C3\%B3gica.pdf

Instituto Nacional de Estadísticas y Censos. Encuesta de Edificaciones, (2016). Materiales predominantes a ser utilizados en la edificación. https:/www.ecuadorencifras.gob.ec/documentos/ web-inec/Estadisticas_Economicas/Encuesta_Edificaciones/2016/2016_EDIFICACIONES_ PRESENTACION.pdf

Lajara, A. (2012). "Estudio comparativo y aplicación de los métodos de evaluación medioambiental BREEAM, LEED y VERDE para materiales y residuos en la construcción sostenible de edificios de oficinas". Proyecto/Trabajo final de carrera. http://hdl.handle.net/2099.1/17250

Leff, E. (2007). "La Complejidad Ambiental". Polis [En línea]. http://journals.openedition.org/polis/4605

López, C; López-Hernández, E. y Ancona I. (2005). "Desarrollo sustentable o sostenible: una definición conceptual". Horizonte Sanitario, vol. 4, núm. 2. https://www.redalyc.org/ pdf/4578/457845044002.pdf

Macías, M. y García J. (2010). "Metodología y herramienta VERDE para la evaluación de la sostenibilidad en edificios". Informes de la Construcción Vol. 62, 517, 87-100. http://oa.upm. es/8477/1/INVE_MEM_2010_83244.pdf

Mercader, M.; Ramírez de Arellano, A. y Olivares, M.(2010). "Cuantificación de los recursos consumidos y emisiones de $\mathrm{CO}_{2}$ producidas en las construcciones de Andalucía y sus implicaciones en el protocolo de Kioto". Tomo I. https://idus.us.es/bitstream/handle/11441/15660/0_Tesis_MPMM-1.pdf?sequence=-

Mercader, M.; Ramírez de Arellano, A. y Olivares, M. (2012). "Modelo de cuantificación de las emisiones de $\mathrm{CO}_{2}$ producidas en edificación derivadas de los recursos materiales consumidos en su ejecución". Informes de la Construcción, Vol 64, No 527 http://informesdelaconstruccion.revistas.csic.es/index.php/informesdelaconstruccion/article/view/2184 
Ministerio de Desarrollo Urbano y Vivienda. (sf). Capítulos de la NEC (Norma Ecuatoriana de la Construcción). https://www.habitatyvivienda.gob.ec/documentos-normativos-nec-norma-ecuatoriana-de-la-construccion/

Ministerio del Ambiente y Agua. https://www.ambiente.gob.ed

Morató, J.; Jiménez, L. y Tollin, N. (2017). "Situación y evolución de la Economía Circular en España Fundación COTEC para la Innovación". https://www.quimicaysociedad.org/libros/situacion-y-evolucion-de-la-economia-circular-en-espana/

Morillón, D. y Hernández, F. (2011). "Metodología para evaluar la sustentabilidad de los materiales de construcción". Universidad Nacional Autónoma de México. https://www.researchgate. net/publication/322369357_Metodologia_para_evaluar_la_sustentabilidad_de_los_ materiales_de_construccion

Morin, E. (1996). "El pensamiento ecologizado". Gazeta de Antropología, 12 (01). http://www.ugr. es/ pwlac/G12_01Edgar_Morin.html

Orellana, V. (2017). "Adobe, puesta en valor y estrategias para la Conservación de una cultura constructiva". Trabajo previo a la obtención del título de Magíster en Conservación y Gestión del Patrimonio Cultural Edificado. Universidad de Cuenca. http://dspace.ucuenca.edu.ec/ handle/123456789/28182

Paternoster, A. (2011). "Herramientas para medir la sostenibilidad corporativa. Un análisis comparativo de las memorias de sostenibilidad". Universitat Politécnica de Catalunya. https:// upcommons.upc.edu/bitstream/handle/2099.1/13644/PFM_Agustin_Paternoster.pd$\mathrm{f}$ ? sequence $=1$ \&isAllowed $=\mathrm{y}$

Peluffo, P. ( 2011). "Análisis de la energía incorporada de un edificio en altura en Uruguay". Tesis para la obtención de título de Master en Construcción de Obras de Arquitectura. Universidad Federal de Río Grande do Sul. https://lume.ufrgs.br/handle/10183/105078

Serrano, A.; Quesada, F.; López, M. ; Guillen, V. y Orellana, D. (2015). "Sobre la evaluación de la sostenibilidad de materiales de construcción". Arte y Sociedad Revista de Investigación, 9. http://asri.eumed.net/9/eco-etiquetas.pdf

Quesada, J.; Calle, A.; Guillén, V.; Ortiz, J. y Lema, K. (2018). "Método de Evaluación Sustentable de la Vivienda en la Ciudad de Cuenca, Ecuador". Revista Técnica "energía". No. 14, Pp. 204212.https://www.researchgate.net/publication/324262453_Metodo_de_Evaluacion_ Sustentable_de_la_Vivienda_en_la_Ciudad_de_Cuenca_Ecuador

Wackernagel M. y Rees W. (1996). "Nuestra Huella Ecológica. Reduciendo el impacto humano sobre la Tierra". LOM Ediciones. https://books.google.com.ec/books?id=ljpRXhe5pygC\&printsec $=$ frontcover\&hl $=e s \# v=0$ nepage $\& q \& f=f a l s e$

Wadel, G.; Avellaneda, J. y Cuchí, A. (2010). "La sostenibilidad en la arquitectura industrializada: cerrando el ciclo de los materiales". Informes de la Construcción Vol. 62, 517, 37-51. https:// pdfs.semanticscholar.org/ee09/b6f4a55ed637a994e3c39cbd1a24bcd8a1f8.pdf 